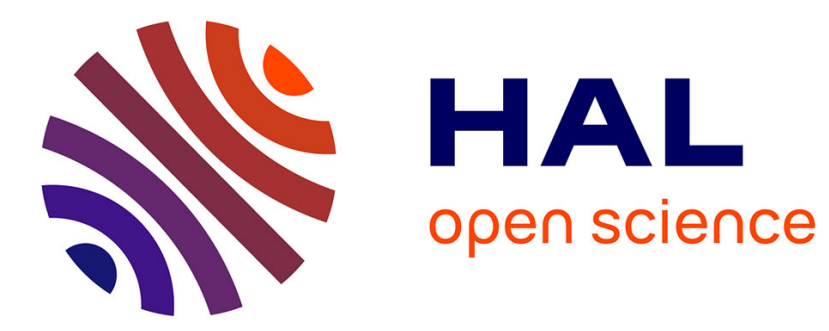

\title{
2D analytical modeling of a wholly superconducting synchronous reluctance motor
}

Gaël Malé, Thierry Lubin, Smail Mezani, Jean Lévêque

\section{To cite this version:}

Gaël Malé, Thierry Lubin, Smail Mezani, Jean Lévêque. 2D analytical modeling of a wholly superconducting synchronous reluctance motor. Superconductor Science and Technology, 2011, 24 (3), pp.1-13. 10.1088/0953-2048/24/3/035014 . hal-00558545

\section{HAL Id: hal-00558545 \\ https://hal.science/hal-00558545}

Submitted on 13 Nov 2011

HAL is a multi-disciplinary open access archive for the deposit and dissemination of scientific research documents, whether they are published or not. The documents may come from teaching and research institutions in France or abroad, or from public or private research centers.
L'archive ouverte pluridisciplinaire HAL, est destinée au dépôt et à la diffusion de documents scientifiques de niveau recherche, publiés ou non, émanant des établissements d'enseignement et de recherche français ou étrangers, des laboratoires publics ou privés. 


\title{
2D Analytical Modeling of a Wholly Superconducting Synchronous Reluctance Motor
}

\author{
Malé G, Lubin T, Mezani $\mathbf{S}$ and Lévèque $\mathbf{J}$ \\ Groupe de Recherche en Electrotechnique et Electronique de Nancy, Henri Poincaré University, Nancy, FRANCE \\ E-mail: gael.male@green.uhp-nancy.fr (G Malé)
}

\begin{abstract}
An analytical computation of the magnetic field distribution in a wholly superconducting synchronous reluctance motor is proposed. The stator of the studied motor consists of three-phase HTS armature windings fed by AC currents. The rotor is made with HTS bulks which present the specificity to have a diamagnetic behavior under zerofield cooling. The electromagnetic torque is obtained by the interaction between the rotating magnetic field created by the HTS windings and the HTS Bulks. The proposed analytical model is based on the resolution of Laplace's and Poisson's equations (by the separation of variables technique) for each sub-domain, i.e. stator windings, air-gap, holes between HTS bulks and exterior iron shield. For the study, the HTS bulks are considered as perfect diamagnetic materials. The boundary and continuity conditions between each sub-domains yield to the global solution. Magnetic field distributions and electromagnetic torque obtained by the analytical method are compared with those obtained from finite element analyses.
\end{abstract}

\section{Keywords}

Analytical solution, bulk superconductor, diamagnetism, high magnetic field, reluctance motor.

\section{Introduction}

Hts materials can be used in various types of electrical devices like motors and generators which represent an important part of the superconducting applications [8]. Among them, Superconducting Synchronous Reluctance Motors (ScSynRM) are studied and tested in different companies and research institutes [9-13].

Conventional synchronous reluctance motors (SynRM) with ferromagnetic materials present a simple structure and rugged characteristics. The rotor of such motors consists of magnetic and nonmagnetic materials. The electromagnetic torque is directly determined by the difference between the $d$-axis inductance $L_{d}$ and the $q$ axis inductance $L_{q}$. Many works have been carried out in the field of SynRM rotor design to improve the machine performances by increasing the $d-q$ axes inductance difference $\left(L_{d}-L_{q}\right)$ [3]. Recently, some authors have proposed to replace the nonmagnetic materials by HTS bulks. HTS bulks present a diamagnetic behavior when they are zero-field cooling. This property is used to decrease the $q$-axis inductance of the SynRM and therefore improves the performances of such motors [3]. The topologies of SynRM with HTS bulks proposed in the literature are all based on the maximization of $\left(L_{d}-\right.$ $L_{q}$ ) and require ferromagnetic materials on both the rotor and the stator.

In this paper, we propose an analytical modeling of a new type of ScSynRM without ferromagnetic materials (except for the outer magnetic shield). The proposed motor uses superconducting materials for both the stator and the rotor. Figure 1 shows the structure of the motor considered in the paper. The stator consists of an air-core three-phase HTS armature windings fed by AC currents. The HTS stator windings generate a rotating magnetic field of high amplitude. The rotating magnetic field rotates at an angular velocity related to the electrical current frequency and the number of pole pairs of the winding configuration. The rotating magnetic field interacts with the HTS bulks placed at the rotor (air-core rotor). The electromagnetic torque is obtained by the magnetic field lines deviation in the airgap due to the presence of the rotor HTS bulks working under diamagnetic properties (zero-field cooling). It is well known that if a superconducting bulk is cooled down without applied magnetic field at a temperature below its critical temperature $T_{c}$, a pure superconductor 




Figure 1. Studied HTS reluctance machine $\left(Q=4, p=2, \theta_{0}=0\right)$.

will not allow any magnetic field to freely enter it, names Meissner effect. This implied that a magnetic field applied near this superconductor will be excluded like for a diamagnetic material.

A cryostat is inserted between the stator windings and the outer iron yoke to cool down the whole HTS motor at low temperature with liquid nitrogen $(77 \mathrm{~K})$ or with liquid neon around $30 \mathrm{~K}$ [8]. The outer iron yoke operates like a magnetic shield to limit the magnetic effects on the external environment.

The performances of the proposed reluctance motor would be analyzed by an analytical model presented in this paper. Analytical methods are, in general, less computational time consuming than numerical ones (like finite-element method) and can provide closed-form solutions giving physical insight for designers. So, they are useful tools for first evaluation of electrical motors performances and for design optimization. In the proposed analytical model, the HTS materials are assumed to be perfect (no AC losses in the HTS coils and perfect diamagnetic behavior of the HTS bulks).

The different sections are written like following. Section II describes the problem and the assumptions of the model. The analytical method for magnetic field calculation (in the air-gap, windings and in the holes sub-domains) is given in section III. The electromagnetic torque expression is developed in section IV. The analytical results are then verified with finite-element method in section $\mathrm{V}$.

\section{Problem description and assumptions}

The structure of the studied ScSynRM is shown in figure 1. It consists of a p pole-pairs armature windings and $Q$ HTS bulks placed in the rotor. As can be seen in figure 1 , the rotor contains a ring of $Q$ holes domains between the $Q$ HTS bulks which are difficult to handle in the analytical prediction of the air-gap magnetic field. Analytical approaches for the magnetic field computation in classical ferromagnetic machines using sub-domains method can be found in the literature [4-7]. However, the proposed analytical models relate to ironcored electrical machine and cannot be used for the analysis of the proposed air-cored machine with HTS bulks.

Here, we propose an analytical solution of the magnetic field distribution for the studied HTS motor. The Laplace and Poisson's equations are solved in each subdomain (air-gap, stator windings, exterior iron yoke, and holes between HTS bulks). The solution is obtained using boundary and interface conditions between the different sub-domains.

The geometrical parameters are:

- for the rotor, inner and outer radii of the bulks $R_{1}$ and $R_{2}$ respectively;

- for the three phase windings, the inner and outer radii $R_{3}$ and $R_{4}$ respectively;

- for the magnetic yoke, the inner and outer radii $R_{5}$ and $R_{6}$ respectively;

- for the external domain (air region), the outer radius $R_{7}$.

The winding-opening is $\alpha$. The hole opening angle is $\beta$. The angular position of the $i$-th hole is defined as

$$
\theta_{i}=-\frac{\beta}{2}+\frac{2 i \pi}{Q}+\theta_{0} \text { with } \quad 1 \leq i \leq Q
$$

where $\theta_{0}$ is the angular position of the HTS bulks rotor. All the calculus are made by using the following assumptions:

- End effects are neglected.

- Relative permeability of the outer magnetic yoke is $\mu_{r} \gg 1,\left(\mu_{\mathrm{r}}=100\right.$ has been chosen $)$

- Magnetic yoke is not saturated.

- HTS bulks have radial sides.

- Perfect diamagnetic behavior of the HTS bulks.

The last assumption is equivalent as to impose a Dirichlet condition on the surfaces of the 
superconducting bulks for the magnetic vector potential

$$
A=0
$$

As shown in figure 1, the whole domain is divided into six sub-domains: the rotor shaft sub-domain (region I), the air-gap sub-domain (region II), the windings subdomain (region III), the outer air-gap (for cryostat) subdomain (region IV), the magnetic yoke sub-domain (region $\mathrm{V}$ ), the exterior sub-domain (air region VI), and the $\mathrm{Q}$ holes sub-domains (regions $\mathrm{i}$ ). The $i$-th holes subdomain shape is shown in figure 2. The sub-domains II to VI have annular shapes.

Due to the presence of electrical current in the stator windings, a magnetic vector potential formulation has been chosen in 2D polar coordinates to describe the problem. According to the adopted assumptions, the magnetic vector potential has only one component along the $z$-direction and only depends on the $r$ and $\theta$ coordinates. The notations used in the paper are

$\boldsymbol{A}_{\boldsymbol{I}}=A_{I}(r, \theta) \cdot \boldsymbol{e}_{z} \quad$ for the rotor shaft sub-domain

$\boldsymbol{A}_{i}=A_{i}(r, \theta) \cdot \boldsymbol{e}_{z} \quad$ for the i-th hole sub-domain

$\boldsymbol{A}_{I I}=A_{I I}(r, \theta) \cdot \boldsymbol{e}_{z} \quad$ for the air-gap sub-domain

$\boldsymbol{A}_{I I I}=A_{I I I}(r, \theta) \cdot \boldsymbol{e}_{z} \quad$ for the windings sub-domain

$\boldsymbol{A}_{I V}=A_{I V}(r, \theta) \cdot \boldsymbol{e}_{z}$ for the outer air-gap sub-domain

$A_{V}=A_{V}(r, \theta) \cdot e_{z} \quad$ for the magnetic yoke sub-domain

$\boldsymbol{A}_{V I}=A_{V I}(r, \theta) \cdot \boldsymbol{e}_{z} \quad$ for the exterior sub-domain

\section{Problem formulation and solution}

By separation of variables technique, the solution of Laplace's equation is considered for the holes, the airgaps and the iron sub-domains, and the Poisson's equation for the windings sub-domain. For simplicity and more clarity of the general solutions in the different sub-domains, the following notations are adopted throughout the paper

$$
\begin{aligned}
& \mathrm{P}_{u}\left(R_{v}, R_{w}\right)=\left(\frac{R_{v}}{R_{w}}\right)^{u}+\left(\frac{R_{v}}{R_{w}}\right)^{-u} \\
& E_{u}\left(R_{v}, R_{w}\right)=\left(\frac{R_{v}}{R_{w}}\right)^{u}-\left(\frac{R_{v}}{R_{w}}\right)^{-u}
\end{aligned}
$$

3.1. General Solution of Laplace's Equation in the $i$-th hole sub-domain (regions $i=1$ to $i=Q$ )

Figure 2 shows the $i$-th hole sub-domain and the associated boundary conditions. The Laplace's equation must be solve in a domain of inner radius $R_{l}$ and outer radius $R_{2}$ delimited by the angles $\theta_{i}$ and $\theta_{i}+\beta$.

$$
\frac{\partial^{2} A_{i}}{\partial r^{2}}+\frac{1}{r} \frac{\partial A_{i}}{\partial r}+\frac{1}{r^{2}} \frac{\partial^{2} A_{i}}{\partial \theta^{2}}=0 \text { for }\left\{\begin{array}{l}
R_{1} \leq r \leq R_{2} \\
\theta_{i} \leq \theta \leq \theta_{i}+\beta
\end{array}\right.
$$

Considering a perfect diamagnetic behavior for the HTS bulks, the radial component of the magnetic field at the sides of the HTS bulk is null. The boundary conditions for the $i$-th hole domain are

$$
\left.A_{i}\right|_{\theta=\theta_{i}}=0 \text { and }\left.A_{i}\right|_{\theta=\theta_{i}+\beta}=0
$$

The continuity of the tangential magnetic field between the $i$-th hole and the air-gap sub-domains leads to

$$
\begin{aligned}
& \left.\frac{\partial A_{i}}{\partial r}\right|_{r=R_{1}}=\left.\frac{\partial A_{I}}{\partial r}\right|_{r=R_{1}} \\
& \left.\frac{\partial A_{i}}{\partial r}\right|_{r=R_{2}}=\left.\frac{\partial A_{I I}}{\partial r}\right|_{r=R_{2}}
\end{aligned}
$$

The general solution of (5) can be found by using the method of the separation of variables, the solution can be written as

$$
A_{i}(r, \theta)=R_{i}(r) . \Theta_{i}(\theta)
$$

Equation (9) is substituted into (5) to obtain two ordinary differential equations where $\lambda(\lambda \in \mathfrak{R})$ is the separation constant.

$$
\begin{gathered}
\Theta_{i}^{\prime \prime}-\lambda \cdot \Theta_{i}=0 \\
r^{2} \cdot R_{i}^{\prime \prime}+r \cdot R_{i}^{\prime}+\lambda \cdot R_{i}=0
\end{gathered}
$$

Using (9), the boundary conditions (6) become

$$
\left.\Theta_{i}\right|_{\theta=\theta_{i}}=0 \text { and }\left.\Theta_{i}\right|_{\theta=\theta_{i}+\beta}=0
$$

The problem is to find the values of the parameter $\lambda$ for which nontrivial solutions of (10) satisfying the boundary conditions (12). Solutions are described by a Sturm-Liouville problem.

Under the latest condition, the values of the parameter $\lambda$ are called eigenvalues and the solutions are called eigenfunctions. A general method to compute the eigenvalues and eigenfunctions for a Sturm-Liouville problem is given in [1]. 


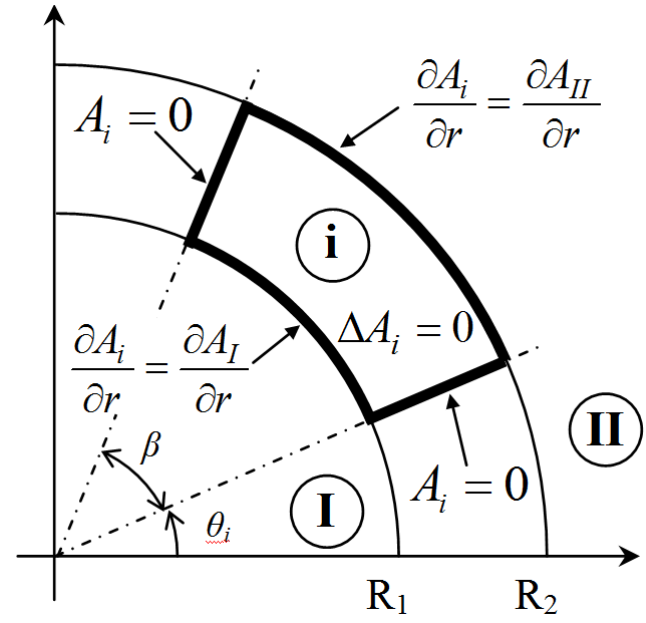

Figure 2. i-th hole sub-domain and its boundary conditions.

The eigenvalues of the problem (10), (12) are

$$
\lambda_{n}=-\left(\frac{n \cdot \pi}{\beta}\right)^{2} \text { with } n=1,2,3, \ldots
$$

The eigenfunctions corresponding to $\lambda_{n}$ are given by

$$
\Theta_{i n}(\theta)=\sin \left(\frac{n \cdot \pi}{\beta}\left(\theta-\theta_{i}\right)\right)
$$

For $\lambda_{n}$, the general solutions of (11) can be written as

$$
R_{i n}(\theta)=C_{n}^{i} \cdot r^{-\frac{n \pi}{\beta}}+D_{n}^{i} \cdot r^{\frac{n \pi}{\beta}}
$$

where $C_{n}^{i}$ and $D_{n}^{i}$ are arbitrary constants.

Writing the general solution as a linear combination of the previous solutions, we have

$$
A_{i}(r, \theta)=\sum_{n=1}^{\infty} \Theta_{i n}(\theta) \cdot R_{i n}(r)
$$

therefore

$$
A_{i}(r, \theta)=\sum_{n=1}^{\infty}\left(C_{n}^{i} \cdot r^{-\frac{n \pi}{\beta}}+D_{n}^{i} \cdot r^{\frac{n \pi}{\beta}}\right) \cdot \sin \left(\frac{n \pi}{\beta}\left(\theta-\theta_{i}\right)\right)
$$

where $C_{n}^{i}$ and $D_{n}^{i}$ are constants which will be determinate by the interface conditions.

Considering the interface conditions (7) and (8), the general solution of the magnetic vector potential in the $i$ th hole domain is rewritten as

$$
\begin{aligned}
& A_{i}(r, \theta)=\sum_{n=1}^{\infty}\left(C_{n}^{i} \frac{\beta \cdot R_{1}}{n \pi} \cdot \frac{P_{n \pi / \beta}\left(r, R_{2}\right)}{\mathrm{E}_{n \pi / \beta}\left(R_{1}, R_{2}\right)} \cdot \sin \left(\frac{n \pi}{\beta}\left(\theta-\theta_{i}\right)\right)\right) \\
& -\sum_{n=1}^{\infty}\left(D_{n}^{i} \frac{\beta \cdot R_{2}}{n \pi} \cdot \frac{P_{n \pi / \beta}\left(r, R_{1}\right)}{\mathrm{E}_{n \pi / \beta}\left(R_{2}, R_{1}\right)} \cdot \sin \left(\frac{n \pi}{\beta}\left(\theta-\theta_{i}\right)\right)\right)
\end{aligned}
$$

where $n$ is a positive integer, $P_{n \pi / \beta}\left(R_{v}, R_{w}\right)$ and $\mathrm{E}_{n \pi / \beta}\left(R_{v}, R_{w}\right)$ are defined by (3) and (4) respectively.

The constants $C_{n}^{i}$ and $D_{n}^{i}$ are determined using a Fourier series expansion of $\left.\frac{\partial A_{I}}{\partial r}\right|_{R_{1}}$ and $\left.\frac{\partial A_{I I}}{\partial r}\right|_{R_{2}}$ over the hole-opening interval $\left[\theta_{i}, \theta_{i}+\beta\right]$

$$
\begin{aligned}
& C_{n}^{i}=\left.\frac{2}{\beta} \int_{\theta_{i}}^{\theta_{i}+\beta} \frac{\partial A_{I}}{\partial r}\right|_{R_{1}} \cdot \sin \left(\frac{n \pi}{\beta}\left(\theta-\theta_{i}\right)\right) \cdot d \theta \\
& D_{n}^{i}=\left.\frac{2}{\beta} \int_{\theta_{i}}^{\theta_{i}+\beta} \frac{\partial A_{I I}}{\partial r}\right|_{R_{2}} \cdot \sin \left(\frac{n \pi}{\beta}\left(\theta-\theta_{i}\right)\right) \cdot d \theta
\end{aligned}
$$

The expressions for the coefficients $C_{n}^{i}$ and $D_{n}^{i}$ are given in the appendix.

\subsection{General solution of Laplace's equation in the Rotor Shaft sub-domain (region I)}

The rotor shaft sub-domain and the associated boundary conditions are shown in figure 3 . The problem to solve is

$$
\frac{\partial^{2} A_{I}}{\partial r^{2}}+\frac{1}{r} \frac{\partial A_{I}}{\partial r}+\frac{1}{r^{2}} \frac{\partial^{2} A_{I}}{\partial \theta^{2}}=0 \text { for }\left\{\begin{array}{l}
0 \leq r \leq R_{1} \\
0 \leq \theta \leq 2 \pi
\end{array}\right.
$$

The boundary condition at the radius $r=R_{1}$ is difficult to handle because of the existence of the holes as shown in figure 1.

Considering the continuity of the magnetic vector potential at the interface between the holes and the rotor shaft domains and considering that the magnetic vector potential is equal to zero elsewhere (diamagnetic proprieties of superconducting bulks), the boundary condition at $r=R_{1}$ can be written as

$$
A_{I}\left(R_{1}, \theta\right)=F(\theta)
$$

with

$$
F(\theta)= \begin{cases}A_{i}\left(R_{1}, \theta\right) & \forall \theta \in\left[\theta_{\mathrm{i}}, \theta_{i}+\beta\right] \\ 0 & \text { elsewhere }\end{cases}
$$




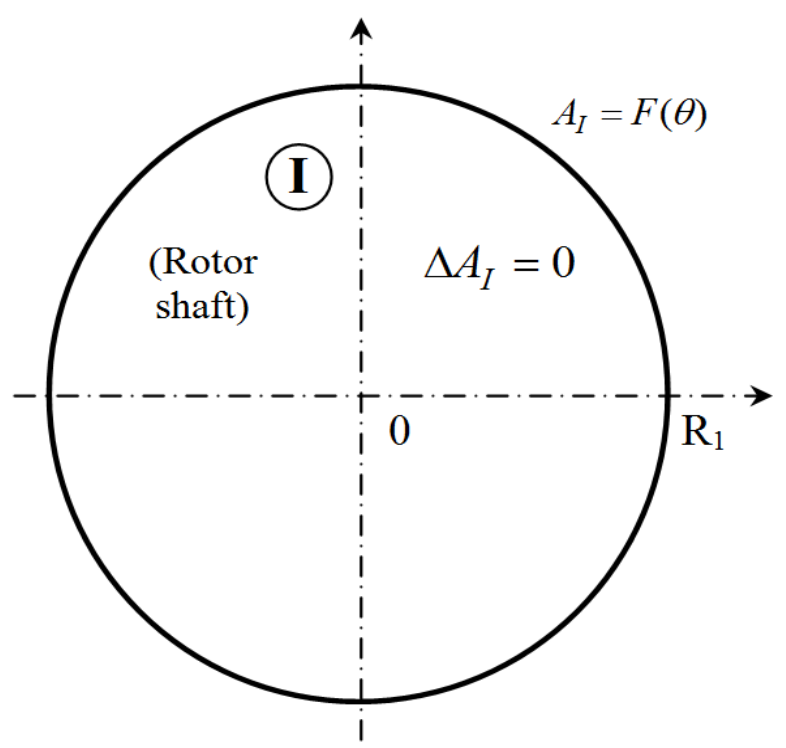

Figure 3. Rotor shaft sub-domain (region I) with its boundary conditions.

where $A_{i}(r, \theta)$ is the magnetic vector potential in the $i$-th hole given by (18).

The distribution of $F(\theta)$ at $r=R_{1}$ along the rotor shaft interval $[0,2 \pi]$ is schematically shown in figure 4 .

The general solution of (21) is well known [1] (periodic Sturm-Liouville problem in a disc). By taking into account the boundary condition (22) and the fact that the magnetic vector potential must be finite at $r=0$, the general solution of (21) can be written as

$$
A_{I}(r, \theta)=A_{0}^{I}+\sum_{h=1}^{\infty}\left(\left(\frac{r}{R_{1}}\right)^{h}\left(A_{h}^{I} \cdot \cos (h \theta)+C_{h}^{I} \cdot \sin (h \theta)\right)\right)
$$

where $h$ is a positive integer. The coefficients $A_{0}^{I}, A_{h}^{I}$ and $C_{h}^{I}$ are determined using a Fourier series expansion of $F(\theta)$ over the interval $[0,2 \pi]$

$$
\begin{gathered}
A_{0}^{I}=\frac{1}{2 \pi} \int_{0}^{2 \pi} F(\theta) \cdot d \theta \\
A_{h}^{I}=\frac{2}{2 \pi} \int_{0}^{2 \pi} F(\theta) \cdot \cos (h \theta) \cdot d \theta \\
C_{h}^{I}=\frac{2}{2 \pi} \int_{0}^{2 \pi} F(\theta) \cdot \sin (h \theta) \cdot d \theta
\end{gathered}
$$

Taking into account (23), the coefficients (25) to (27) can be rewritten as

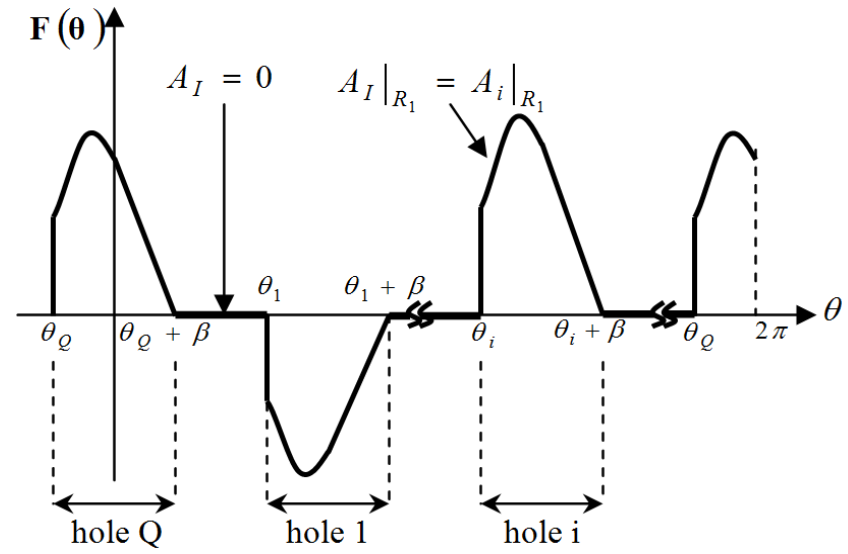

Figure 4. Schematic representation of $\mathrm{F}(\theta)$ at $r=R_{l}$.

$$
\begin{gathered}
A_{0}^{I}=\frac{1}{2 \pi} \sum_{i=1}^{Q} \int_{\theta_{i}}^{\theta_{i}+\beta} A_{i}\left(R_{1}, \theta\right) \cdot d \theta \\
A_{h}^{I}=\frac{2}{2 \pi} \sum_{i=1}^{Q} \int_{\theta_{i}}^{\theta_{i}+\beta} A_{i}\left(R_{1}, \theta\right) \cdot \cos (h \theta) \cdot d \theta \\
C_{h}^{I}=\frac{2}{2 \pi} \sum_{i=1}^{Q} \int_{\theta_{i}}^{\theta_{i}+\beta} A_{i}\left(R_{1}, \theta\right) \cdot \sin (h \theta) \cdot d \theta
\end{gathered}
$$

where $Q$ is the number of HTS bulks in the rotor. It is worth noting that the mutual interaction between the holes is related by the sum operation on $Q$ in (28), (29) and (30). The expressions for the coefficients $A_{0}^{I}, A_{h}^{I}$ and $C_{h}^{I}$ are given in the appendix.

3.3. General solution of Laplace's equation in the airgap sub-domain (region II)

The air-gap sub-domain and the associated boundary conditions are shown in figure 5 . The problem to solve is

$\frac{\partial^{2} A_{I I}}{\partial r^{2}}+\frac{1}{r} \frac{\partial A_{I I}}{\partial r}+\frac{1}{r^{2}} \frac{\partial^{2} A_{I I}}{\partial \theta^{2}}=0$ for $\left\{\begin{array}{l}R_{2} \leq r \leq R_{3} \\ 0 \leq \theta \leq 2 \pi\end{array}\right.$

The boundary condition at $r=R_{3}$ is

$$
\left.\frac{\partial A_{I I}}{\partial r}\right|_{r=R_{3}}=\left.\frac{\partial A_{I I I}}{\partial r}\right|_{r=R_{3}}
$$

The same method as the one presented in sub-section B is used to determine the boundary condition at $r=R_{2}$. Introducing the function $G(\theta)$, the boundary condition can be written as

$$
A_{I I}\left(R_{2}, \theta\right)=G(\theta)
$$




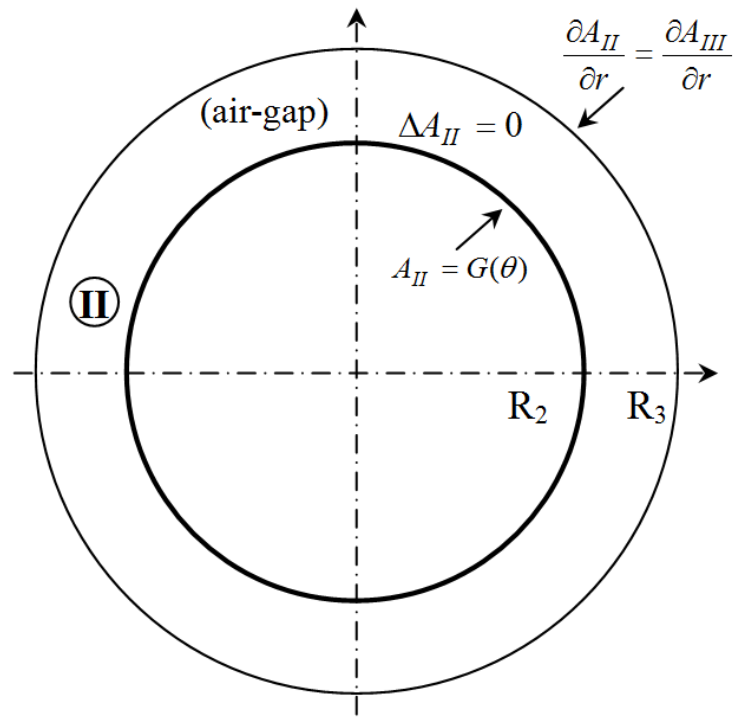

Figure 5. Air-gap sub-domain (region II) and its boundary conditions.

with

$$
G(\theta)= \begin{cases}A_{i}\left(R_{2}, \theta\right) & \forall \theta \in\left[\theta_{\mathrm{i}}, \theta_{i}+\beta\right] \\ 0 & \text { elsewhere }\end{cases}
$$

where $A_{i}(r, \theta)$ is the magnetic vector potential in the $i$ th hole given by (18). The distribution of $G(\theta)$ along the air-gap surface at $r=R_{2}$ presents the same shape as the one presented in figure 4.

The general solution of (31) is well known [1] (periodic Sturm-Liouville problem in an annulus). By taking into account the boundary conditions (32) and (33), the general solution of the magnetic vector potential in the air-gap can be written as

$$
\begin{aligned}
& A_{I I}(r, \theta)=A_{0}^{I I}+B_{0}^{I I} \cdot \ln \left(\frac{R_{2}}{r}\right) \\
& +\sum_{j=1}^{\infty}\left(A_{j}^{I I} \cdot \frac{R_{3}}{j} \cdot \frac{E_{j}\left(r, R_{2}\right)}{P_{j}\left(R_{3}, R_{2}\right)}+B_{j}^{I I} \cdot \frac{P_{j}\left(r, R_{3}\right)}{P_{j}\left(R_{2}, R_{3}\right)}\right) \cdot \cos (j \cdot \theta) \\
& +\sum_{j=1}^{\infty}\left(C_{j}^{I I} \cdot \frac{R_{3}}{j} \cdot \frac{E_{j}\left(r, R_{2}\right)}{P_{j}\left(R_{3}, R_{2}\right)}+D_{j}^{I I} \cdot \frac{P_{j}\left(r, R_{3}\right)}{P_{j}\left(R_{2}, R_{3}\right)}\right) \cdot \sin (j \cdot \theta)
\end{aligned}
$$

where $j$ is a positive integer, $\mathrm{P}_{j}\left(R_{v}, R_{w}\right)$ and $\mathrm{E}_{j}\left(R_{v}, R_{w}\right)$ are defined by (3) and (4). The coefficients $A_{0}^{I I}, B_{0}^{I I}, \quad A_{j}^{I I}, B_{j}^{I I}, \quad C_{j}^{I I} \quad$ and $\quad D_{j}^{I I}$ are determined using Fourier series expansions of $\left.\frac{\partial A_{I I}}{\partial r}\right|_{R_{3}}$ and $G(\theta)$ over the air-gap interval $[0,2 \pi]$

$$
\begin{gathered}
A_{0}^{I I}=\frac{1}{2 \pi} \int_{0}^{2 \pi} G(\theta) \cdot d \theta \\
B_{0}^{I I}=\left.\frac{R_{3}}{2 \pi} \int_{0}^{2 \pi} \frac{\partial A_{I I I}}{\partial \theta}\right|_{R_{3}} \cdot d \theta \\
A_{j}^{I I}=\left.\frac{2}{2 \pi} \int_{0}^{2 \pi} \frac{\partial A_{I I I}}{\partial \theta}\right|_{R_{3}} \cdot \cos (j \theta) \cdot d \theta \\
B_{j}^{I I}=\frac{2}{2 \pi} \int_{0}^{2 \pi} G(\theta) \cdot \cos (j \theta) \cdot d \theta \\
C_{j}^{I I}=\left.\frac{2}{2 \pi} \int_{0}^{2 \pi} \frac{\partial A_{I I I}}{\partial \theta}\right|_{R_{3}} \cdot \sin (j \theta) \cdot d \theta \\
D_{j}^{I I}=\frac{2}{2 \pi} \int_{0}^{2 \pi} G(\theta) \cdot \sin (j \theta) \cdot d \theta
\end{gathered}
$$

The expressions for the coefficients $A_{0}^{I I}, B_{0}^{I I}, A_{j}^{I I}, B_{j}^{I I}$, $C_{j}^{I I}$ and $D_{j}^{I I}$ are given in the appendix.

The flux density distribution in the air-gap can be deduced from the magnetic vector potential by

$$
\begin{aligned}
& B_{I I r}=\frac{1}{r} \frac{\partial A_{I I}}{\partial \theta} \\
& B_{I I \theta}=-\frac{\partial A_{I I}}{\partial r}
\end{aligned}
$$

The radial and the tangential components of the magnetic flux in the air-gap are then

$$
\begin{aligned}
& B_{I I r}(r, \theta)= \\
& -\sum_{j=1}^{\infty}\left(A_{j}^{I I} \cdot \frac{R_{3}}{j} \cdot \frac{E_{j}\left(r, R_{2}\right)}{P_{j}\left(R_{3}, R_{2}\right)}+B_{j}^{I I} \cdot \frac{P_{j}\left(r, R_{3}\right)}{P_{j}\left(R_{2}, R_{3}\right)}\right) \cdot\left(\frac{j}{r}\right) \cdot \sin (j \cdot \theta) \\
& +\sum_{j=1}^{\infty}\left(C_{j}^{I I} \cdot \frac{R_{3}}{j} \cdot \frac{E_{j}\left(r, R_{2}\right)}{P_{j}\left(R_{3}, R_{2}\right)}+D_{j}^{I I} \cdot \frac{P_{j}\left(r, R_{3}\right)}{P_{j}\left(R_{2}, R_{3}\right)}\right) \cdot\left(\frac{j}{r}\right) \cdot \cos (j \cdot \theta)
\end{aligned}
$$

$$
B_{I I \theta}(r, \theta)=-\frac{B_{0}^{I I}}{r}
$$

$-\sum_{j=1}^{\infty}\left(\frac{j}{r}\right) \cdot\left(A_{j}^{I I} \frac{R_{3}}{j} \frac{P_{j}\left(r, R_{2}\right)}{P_{j}\left(R_{3}, R_{2}\right)}+B_{j}^{I I} \frac{E_{j}\left(r, R_{3}\right)}{P_{j}\left(R_{2}, R_{3}\right)}\right) \cdot \cos (j \cdot \theta)$ $-\sum_{j=1}^{\infty}\left(\frac{j}{r}\right) \cdot\left(C_{j}^{I I} \frac{R_{3}}{j} \frac{P_{j}\left(r, R_{2}\right)}{P_{j}\left(R_{3}, R_{2}\right)}+D_{j}^{I I} \frac{E_{j}\left(r, R_{3}\right)}{P_{j}\left(R_{2}, R_{3}\right)}\right) \cdot \sin (j \cdot \theta)$ 
3.4. General solution of Laplace's equation in the outer air-gap sub-domain (region IV)

The outer air-gap sub-domain and the associated boundary conditions are shown in figure 6 . The problem to solve is

$$
\frac{\partial^{2} A_{I V}}{\partial r^{2}}+\frac{1}{r} \frac{\partial A_{I V}}{\partial r}+\frac{1}{r^{2}} \frac{\partial^{2} A_{I V}}{\partial \theta^{2}}=0 \text { for }\left\{\begin{array}{l}
R_{4} \leq r \leq R_{5} \\
0 \leq \theta \leq 2 \pi
\end{array}\right.
$$

The boundary condition at the radius $r=R_{4}$ can be written as

$$
\left.\frac{\partial A_{I V}}{\partial r}\right|_{r=R_{4}}=\left.\frac{\partial A_{I I I}}{\partial r}\right|_{r=R_{4}}
$$

The boundary condition at $r=R_{5}$ is

$$
A_{I V}\left(R_{5}, \theta\right)=A_{V}\left(R_{5}, \theta\right)
$$

By taking into account the boundary conditions (47) and (48), the general solution of the magnetic vector potential in the outer air-gap sub-domain can be written as

$$
\begin{aligned}
& A_{I V}(r, \theta)=A_{0}^{I V}+B_{0}^{I V} \cdot \ln \left(\frac{R_{5}}{r}\right) \\
& +\sum_{l=1}^{\infty}\left(A_{l}^{I V} \cdot \frac{R_{4}}{l} \cdot \frac{E_{l}\left(r, R_{5}\right)}{P_{l}\left(R_{4}, R_{5}\right)}+B_{l}^{I V} \cdot \frac{P_{l}\left(r, R_{4}\right)}{P_{l}\left(R_{5}, R_{4}\right)}\right) \cdot \cos (l . \theta) \\
& +\sum_{l=1}^{\infty}\left(C_{l}^{I V} \cdot \frac{R_{4}}{l} \cdot \frac{E_{l}\left(r, R_{5}\right)}{P_{l}\left(R_{4}, R_{5}\right)}+D_{l}^{I V} \cdot \frac{P_{l}\left(r, R_{4}\right)}{P_{l}\left(R_{5}, R_{4}\right)}\right) \cdot \sin (l \cdot \theta)
\end{aligned}
$$

where $l$ is a positive integer.

The coefficients $A_{0}^{I V}, B_{0}^{I V}, A_{l}^{I V}, B_{l}^{I V}, C_{l}^{I V}$ and $D_{l}^{I V}$ are determined by Fourier series expansions of $\left.\frac{\partial A_{I V}}{\partial r}\right|_{R_{4}}$ and $A_{I V}\left(R_{5}, \theta\right)$ over the interval $[0,2 \pi]$

$$
\begin{gathered}
A_{0}^{I V}=\frac{1}{2 \pi} \int_{0}^{2 \pi} A_{V}\left(R_{5}, \theta\right) \cdot d \theta \\
B_{0}^{I V}=\left.\frac{R_{4}}{2 \pi} \int_{0}^{2 \pi} \frac{\partial A_{I I I}}{\partial \theta}\right|_{R_{4}} \cdot d \theta \\
A_{l}^{I V}=\left.\frac{2}{2 \pi} \int_{0}^{2 \pi} \frac{\partial A_{I I I}}{\partial \theta}\right|_{R_{4}} \cdot \cos (l \theta) \cdot d \theta \\
B_{l}^{I V}=\frac{2}{2 \pi} \int_{0}^{2 \pi} A_{V}\left(R_{5}, \theta\right) \cdot \cos (l \theta) \cdot d \theta
\end{gathered}
$$

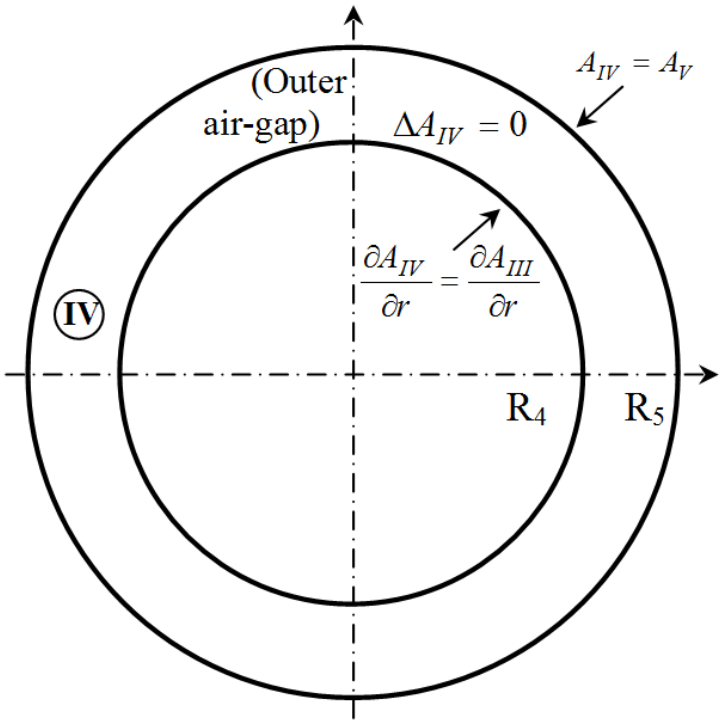

Figure 6. Outer air-gap sub-domain (region IV) with its boundary conditions.

$$
\begin{aligned}
& C_{l}^{I V}=\left.\frac{2}{2 \pi} \int_{0}^{2 \pi} \frac{\partial A_{I I I}}{\partial \theta}\right|_{R_{4}} \cdot \sin (l \theta) \cdot d \theta \\
& D_{l}^{I V}=\frac{2}{2 \pi} \int_{0}^{2 \pi} A_{V}\left(R_{5}, \theta\right) \cdot \sin (l \theta) \cdot d \theta
\end{aligned}
$$

The expressions for the coefficients $A_{0}^{I V}, B_{0}^{I V}, A_{l}^{I V}$, $B_{l}^{I V}, C_{l}^{I V}$ and $D_{l}^{I V}$ are given in the appendix.

3.5. General solution of Laplace's equation in the magnetic yoke sub-domain (region $V$ )

The magnetic yoke sub-domain and the associated boundary conditions are shown in figure 7 . The problem to solve is

$\frac{\partial^{2} A_{V}}{\partial r^{2}}+\frac{1}{r} \frac{\partial A_{V}}{\partial r}+\frac{1}{r^{2}} \frac{\partial^{2} A_{V}}{\partial \theta^{2}}=0$ for $\left\{\begin{array}{l}R_{5} \leq r \leq R_{6} \\ 0 \leq \theta \leq 2 \pi\end{array}\right.$

Considering the continuity of the tangential component of the magnetic field at the radius $r=R_{5}$, The boundary condition can be written as

$$
\left.\frac{1}{\mu_{0} \cdot \mu_{r}} \cdot \frac{d A_{V}}{d r}\right|_{r=R_{5}}=\left.\frac{1}{\mu_{0}} \cdot \frac{d A_{I V}}{d r}\right|_{r=R_{5}}
$$

where $\mu_{0}$ is the permeability of the vacuum and $\mu_{r}$ is the relative permeability of the iron.

The boundary condition at $r=R_{6}$ is

$$
A_{V}\left(R_{6}, \theta\right)=A_{V I}\left(R_{6}, \theta\right)
$$




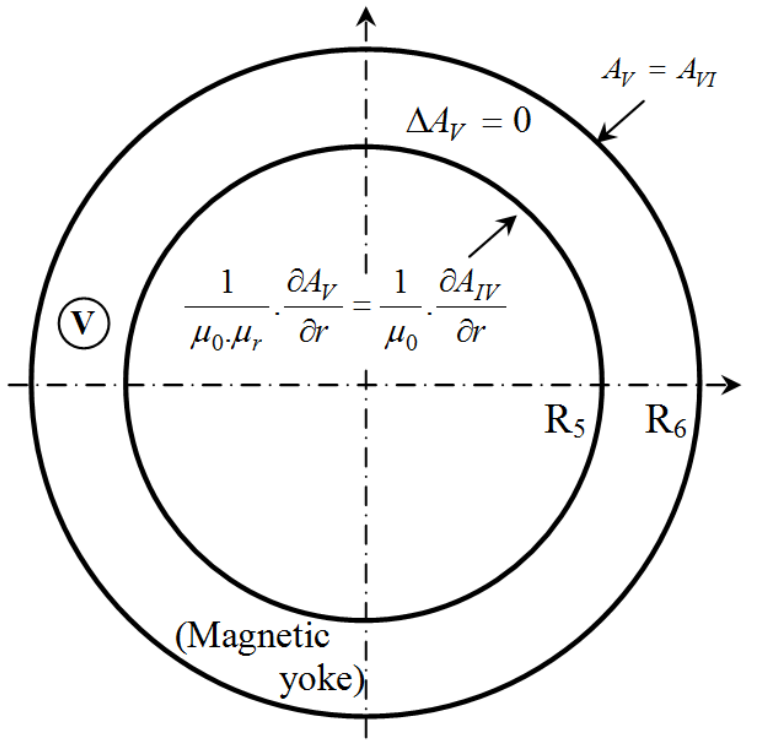

Figure 7. Magnetic yoke sub-domain (region V) with its boundary conditions.

By taking into account the boundary conditions (57) and (58), the general solution of the magnetic vector potential in the magnetic yoke sub-domain can be written as

$$
\begin{aligned}
& A_{V}(r, \theta)=A_{0}^{V}+B_{0}^{V} \cdot \ln \left(\frac{R_{6}}{r}\right) \\
& +\sum_{f=1}^{\infty}\left(A_{f}^{V} \frac{R_{5}}{f} \cdot \frac{E_{f}\left(r, R_{6}\right)}{P_{f}\left(R_{5}, R_{6}\right)}+B_{f}^{V} \frac{P_{f}\left(r, R_{5}\right)}{P_{f}\left(R_{6}, R_{5}\right)}\right) \cdot \cos (f \cdot \theta) \\
& +\sum_{f=1}^{\infty}\left(C_{f}^{V} \frac{R_{5}}{f} \cdot \frac{E_{f}\left(r, R_{6}\right)}{P_{f}\left(R_{5}, R_{6}\right)}+D_{f}^{V} \frac{P_{f}\left(r, R_{5}\right)}{P_{f}\left(R_{6}, R_{5}\right)}\right) \cdot \sin (f \cdot \theta)
\end{aligned}
$$

where $f$ is a positive integer, the coefficients $A_{0}^{V}, B_{0}^{V}$, $A_{f}^{V}, B_{f}^{V}, C_{f}^{V}$ and $D_{f}^{V}$ are determined by Fourier series expansions of $\left.\frac{\partial A_{V}}{\partial r}\right|_{R_{5}}$ and $A_{I V}\left(R_{6}, \theta\right)$ over the interval $[0,2 \pi]$

$$
\begin{gathered}
A_{0}^{V}=\frac{1}{2 \pi} \int_{0}^{2 \pi} A_{V I}\left(R_{6}, \theta\right) \cdot d \theta \\
B_{0}^{V}=\left.\frac{R_{5}}{2 \pi} \int_{0}^{2 \pi} \mu_{r} \cdot \frac{\partial A_{I V}}{\partial \theta}\right|_{R_{5}} \cdot d \theta \\
A_{f}^{V}=\left.\frac{2}{2 \pi} \int_{0}^{2 \pi} \mu_{r} \cdot \frac{\partial A_{I V}}{\partial \theta}\right|_{R_{5}} \cdot \cos (f . \theta) \cdot d \theta
\end{gathered}
$$

$$
\begin{gathered}
B_{f}^{V}=\frac{2}{2 \pi} \int_{0}^{2 \pi} A_{V I}\left(R_{6}, \theta\right) \cdot \cos (f . \theta) \cdot d \theta \\
C_{f}^{V}=\left.\frac{2}{2 \pi} \int_{0}^{2 \pi} \mu_{r} \cdot \frac{\partial A_{I V}}{\partial \theta}\right|_{R_{5}} \cdot \sin (f . \theta) \cdot d \theta \\
D_{f}^{V}=\frac{2}{2 \pi} \int_{0}^{2 \pi} A_{V I}\left(R_{6}, \theta\right) \cdot \sin (f . \theta) \cdot d \theta
\end{gathered}
$$

The expressions for the coefficients $A_{0}^{V}, B_{0}^{V}, A_{f}^{V}, B_{f}^{V}, C_{f}^{V}$ and $D_{f}^{V}$ are given in the appendix.

3.6. General solution of Laplace's equation in the exterior sub-domain (region VI)

The exterior sub-domain and the associated boundary conditions are shown in figure 8 . The problem to solve is

$$
\frac{\partial^{2} A_{V I}}{\partial r^{2}}+\frac{1}{r} \frac{\partial A_{V I}}{\partial r}+\frac{1}{r^{2}} \frac{\partial^{2} A_{V I}}{\partial \theta^{2}}=0 \text { for }\left\{\begin{array}{l}
R_{6} \leq r \leq R_{7} \\
0 \leq \theta \leq 2 \pi
\end{array}\right.
$$

The boundary condition for the exterior domain at $r=R_{6}$ can be written as

$$
\left.\frac{1}{\mu_{0}} \cdot \frac{\partial A_{V I}}{\partial r}\right|_{r=R_{6}}=\left.\frac{1}{\mu_{0} \cdot \mu_{r}} \cdot \frac{\partial A_{V}}{\partial r}\right|_{r=R_{6}}
$$

The boundary condition for the exterior sub-domain at $r=R_{7}$ is

$$
A_{V I}\left(R_{7}, \theta\right)=0
$$

The radius $R_{7}$ is chosen far enough to not affect the results. By taking into account the boundary conditions (67) and (68), the general solution of the magnetic vector potential in the exterior sub-domain can be written as

$$
\begin{aligned}
& A_{V I}(r, \theta)=B_{0}^{V I} \cdot \ln \left(\frac{R_{7}}{r}\right) \\
& +\sum_{m=1}^{\infty}\left(\frac{R_{6}}{m} \frac{E_{m}\left(r, R_{7}\right)}{P_{m}\left(R_{6}, R_{7}\right)}\right) \cdot\left(A_{m}^{V I} \cos (m \cdot \theta)+C_{m}^{V I} \sin (m \cdot \theta)\right)
\end{aligned}
$$

where $m$ is a positive integer The coefficients $B_{0}^{V I}, A_{m}^{V I}$ and $C_{m}^{V I}$ are determined by Fourier series expansions of $\left.\frac{\partial A_{V I}}{\partial r}\right|_{R_{6}}$ and $A_{V I}\left(R_{7}, \theta\right)$ over the interval $[0,2 \pi]$ 




Figure 8. Exterior sub-domain (region VI) with its boundary conditions.

$$
\begin{gathered}
B_{0}^{V I}=\left.\frac{R_{6}}{2 \pi} \int_{0}^{2 \pi} \frac{1}{\mu_{f e r}} \cdot \frac{\partial A_{V}}{\partial \theta}\right|_{R_{6}} \cdot d \theta \\
A_{m}^{V I}=\left.\frac{2}{2 \pi} \int_{0}^{2 \pi} \frac{1}{\mu_{f e r}} \cdot \frac{\partial A_{V}}{\partial \theta}\right|_{R_{6}} \cdot \cos (m \cdot \theta) \cdot d \theta \\
C_{m}^{V I}=\left.\frac{2}{2 \pi} \int_{0}^{2 \pi} \frac{1}{\mu_{f e r}} \cdot \frac{\partial A_{V}}{\partial \theta}\right|_{R_{6}} \cdot \sin (m \cdot \theta) \cdot d \theta
\end{gathered}
$$

The expressions for the coefficients $B_{0}^{V I}, A_{m}^{V I}$ and $C_{m}^{V I}$ are given in the appendix.

4. General solution of Poisson's equation in windings sub- domains (regions III)

The windings sub-domain and its associated boundary conditions are shown in figure 9. The problem is the following

$\frac{\partial^{2} A_{I I I}}{\partial r^{2}}+\frac{1}{r} \frac{\partial A_{I I I}}{\partial r}+\frac{1}{r^{2}} \frac{\partial^{2} A_{I I I}}{\partial \theta^{2}}=-\mu_{0} . J$ for $\left\{\begin{array}{l}R_{3} \leq r \leq R_{4} \\ 0 \leq \theta \leq 2 \pi\end{array}\right.$

where $\mu_{0}$ is the permeability of the vacuum and $J$ is the total current density corresponding to the three-phase windings.

The boundary conditions at $r=R_{3}$ and $r=R_{4}$ are respectively

$$
\begin{aligned}
& A_{I I I}\left(R_{3}, \theta\right)=A_{I I}\left(R_{3}, \theta\right) \\
& A_{I I I}\left(R_{4}, \theta\right)=A_{I V}\left(R_{4}, \theta\right)
\end{aligned}
$$

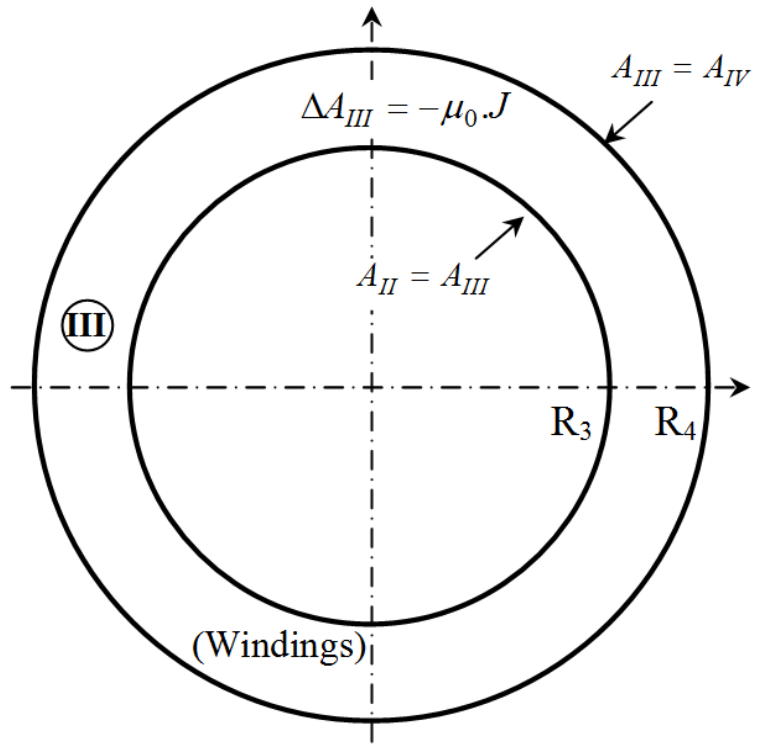

Figure 9. Windings sub-domain (region III) and its boundary conditions.

As shown in figure 1, a three-phase winding is used in the studied motor. Figure 10 shows the total current density distribution along $\theta$-direction in the three-phase windings where $J_{0}$ corresponds to the maximum current density through a winding. The three-phase windings are fed with electrical current such as $I_{a}=I$ and $I_{b}=I_{c}=-I / 2$. The distribution of the total current density $J$ can be expressed in Fourier's series expansion (76) and replaced in (73)

$$
J(\theta)=\sum_{k=1}^{\infty} J_{k} \cdot \cos \left(k \cdot\left(\theta-\varphi_{0}\right)\right)
$$

where $\varphi_{0}$ is the current angle control.

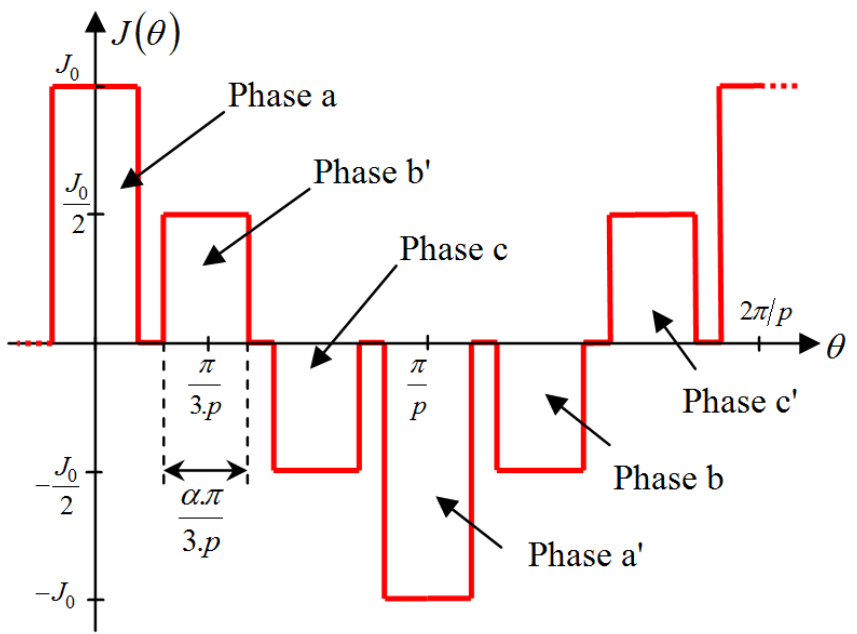

Figure 10. Current density distribution in the threephase windings along $\theta$-direction. 
$A_{I I I}(r, \theta)=A_{0}^{I I I} \cdot \frac{\ln \left(r / R_{4}\right)}{\ln \left(R_{3} / R_{4}\right)}+B_{0}^{I I I} \cdot \frac{\ln \left(r / R_{3}\right)}{\ln \left(R_{4} / R_{3}\right)}$

$+\sum_{k=1}^{\infty}\left(A_{k}^{I I I} \frac{E_{k}\left(r, R_{4}\right)}{E_{k}\left(R_{3}, R_{4}\right)}+B_{k}^{I I I} \frac{E_{k}\left(r, R_{3}\right)}{E_{k}\left(R_{4}, R_{3}\right)}\right) \cdot \cos (k \cdot \theta)$

$+\sum_{k=1}^{\infty} G_{k}(r) \cdot \cos \left(k \cdot \varphi_{0}\right) \cdot \cos (k \cdot \theta)$

$+\sum_{k=1}^{\infty}\left(C_{k}^{I I I} \frac{E_{k}\left(r, R_{4}\right)}{E_{k}\left(R_{3}, R_{4}\right)}+D_{k}^{I I I} \frac{E_{k}\left(r, R_{3}\right)}{E_{k}\left(R_{4}, R_{3}\right)}\right) \cdot \sin (k \cdot \theta)$

$+\sum_{k=1}^{\infty} G_{k}(r) \cdot \sin \left(k \cdot \varphi_{0}\right) \cdot \sin (k \cdot \theta)$

where

$$
\begin{array}{r}
G_{k}(r)=\frac{E_{k}\left(r, R_{4}\right)}{E_{k}\left(R_{3}, R_{4}\right)} \cdot\left(f_{k}\left(R_{4}\right) \cdot\left(\frac{R_{3}}{R_{4}}\right)^{k}-f_{k}\left(R_{3}\right)\right)-\left(f_{k}\left(R_{4}\right) \cdot\left(\frac{r}{R_{4}}\right)^{k}-f_{k}(r)\right) \\
-\frac{E_{k}\left(r, R_{3}\right)}{E_{k}\left(R_{4}, R_{3}\right)} \cdot\left(f_{k}\left(R_{3}\right) \cdot\left(\frac{R_{4}}{R_{3}}\right)^{k}-f_{k}\left(R_{4}\right)\right)-\left(f_{k}\left(R_{3}\right) \cdot\left(\frac{r}{R_{3}}\right)^{k}-f_{k}(r)\right)
\end{array}
$$

and

$$
f_{k}(r)= \begin{cases}\frac{\mu_{0} J_{k} r^{2}}{\left(k^{2}-4\right)} & \text { if } k=i_{k} \cdot p \text { with } i_{k}=1,3,5, \ldots \\ -\frac{\mu_{0} J_{k} r^{2}}{16}(4 \cdot \ln (\mathrm{r})-1) & \text { if } k=2 \\ 0 & \text { otherwise }\end{cases}
$$

where $k$ is a positive integer, $p$ is the number of polepairs of the three phase windings and $E_{k}\left(R_{v}, R_{w}\right)$ is defined by (4).

The coefficients $A_{0}^{I I I}, \quad B_{0}^{I I I}, \quad A_{k}^{I I I}, \quad B_{k}^{I I I}, \quad C_{k}^{I I I}$ and $D_{k}^{I I I}$ are determined using Fourier series expansions of $A_{I I}\left(R_{3}, \theta\right)$ and $A_{I I I}\left(R_{4}, \theta\right)$ over the interval $[0,2 \pi]$

$$
\begin{gathered}
A_{0}^{I I I}=\frac{1}{2 \pi} \int_{0}^{2 \pi} A_{I I}\left(R_{3}, \theta\right) \cdot d \theta \\
B_{0}^{I I I}=\frac{1}{2 \pi} \int_{0}^{2 \pi} A_{I V}\left(R_{4}, \theta\right) \cdot d \theta \\
A_{k}^{I I I}=\frac{2}{2 \pi} \int_{0}^{2 \pi} A_{I I}\left(R_{3}, \theta\right) \cdot \cos (k \cdot \theta) \cdot d \theta \\
B_{k}^{I I I}=\frac{2}{2 \pi} \int_{0}^{2 \pi} A_{I V}\left(R_{4}, \theta\right) \cdot \cos (k \cdot \theta) \cdot d \theta
\end{gathered}
$$

$$
\begin{aligned}
& C_{k}^{I I I}=\frac{2}{2 \pi} \int_{0}^{2 \pi} A_{I I}\left(R_{3}, \theta\right) \cdot \sin (k \cdot \theta) \cdot d \theta \\
& D_{k}^{I I I}=\frac{2}{2 \pi} \int_{0}^{2 \pi} A_{I V}\left(R_{4}, \theta\right) \cdot \sin (k \cdot \theta) \cdot d \theta
\end{aligned}
$$

The expressions for the coefficients $A_{0}^{I I I}, B_{0}^{I I I}, A_{k}^{I I I}$, $B_{k}^{I I I}, C_{k}^{I I I}$ and $D_{k}^{I I I}$ are given in the appendix.

\section{Electromagnetic torque calculation}

The electromagnetic torque is obtained using the Maxwell stress tensor. A circle of radius $R_{e}$ in the airgap sub-domain is taken as the integration path so the electromagnetic torque is expressed as follows

$$
\Gamma_{e}=\frac{L \cdot R_{e}^{2}}{\mu_{0}} \cdot \int_{0}^{2 \pi} B_{I I r}\left(R_{e}, \theta\right) \cdot B_{I I \theta}\left(R_{e}, \theta\right) \cdot d \theta
$$

where $L$ is the axial length of the superconducting reluctance motor. According to (44) and (45), the analytical expression for the electromagnetic torque becomes

$$
\Gamma_{e}=-\frac{\pi \cdot L}{\mu_{0}} \sum_{j=1}^{\infty} j^{2} \cdot\left(B_{n 1}^{j} \cdot H_{t 2}^{j}-B_{n 2}^{j} \cdot H_{t 1}^{j}\right)
$$

where

$$
\begin{aligned}
& B_{n 1}^{j}=A_{j}^{I I} \cdot \frac{R_{3}}{j} \cdot \frac{E_{j}\left(R_{e}, R_{2}\right)}{P_{j}\left(R_{3}, R_{2}\right)}+B_{j}^{I I} \cdot \frac{\mathrm{P}_{j}\left(R_{e}, R_{3}\right)}{P_{j}\left(R_{2}, R_{3}\right)} \\
& B_{n 2}^{j}=C_{j}^{I I} \cdot \frac{R_{3}}{j} \cdot \frac{E_{j}\left(R_{e}, R_{2}\right)}{P_{j}\left(R_{3}, R_{2}\right)}+D_{j}^{I I} \cdot \frac{\mathrm{P}_{j}\left(R_{e}, R_{3}\right)}{P_{j}\left(R_{2}, R_{3}\right)} \\
& H_{t 1}^{j}=A_{j}^{I I} \cdot \frac{R_{3}}{j} \cdot \frac{P_{j}\left(R_{e}, R_{2}\right)}{P_{j}\left(R_{3}, R_{2}\right)}+B_{j}^{I I} \cdot \frac{E_{j}\left(R_{e}, R_{3}\right)}{P_{j}\left(R_{2}, R_{3}\right)} \\
& H_{t 2}^{j}=C_{j}^{I I} \cdot \frac{R_{3}}{j} \cdot \frac{P_{j}\left(R_{e}, R_{2}\right)}{P_{j}\left(R_{3}, R_{2}\right)}+D_{j}^{I I} \cdot \frac{E_{j}\left(R_{e}, R_{3}\right)}{P_{j}\left(R_{2}, R_{3}\right)}
\end{aligned}
$$

\section{6. results and comparison with finite element}

In order to validate the proposed analytical model, a comparison with 2D finite element simulations has been realized using FEMM software [2]. The geometrical parameters of the studied HTS reluctance motor are given in table 1. The geometrical parameters have been chosen arbitrary. The main objective of this paper is to show the accuracy of the proposed analytical model. The analytical solutions in the hole sub-domain and in the other sub-domains have been computed with a finite number of harmonic terms $N$ and $K$ as indicated in table 1. 
Table 1. Parameters of the model.

\begin{tabular}{|c|c|c|}
\hline Symbol & Quantity & Value \\
\hline $\mathrm{R}_{1}$ & Inner radius of bulks & $9 \mathrm{~cm}$ \\
\hline $\mathrm{R}_{2}$ & Outer radius of bulks & $9.5 \mathrm{~cm}$ \\
\hline $\mathrm{R}_{3}$ & Inner radius of the windings & $10 \mathrm{~cm}$ \\
\hline $\mathrm{R}_{4}$ & Outer radius of the windings & $12.5 \mathrm{~cm}$ \\
\hline $\mathrm{R}_{5}$ & $\begin{array}{l}\text { Outer radius of the outer air- } \\
\text { gap (cryostat yoke) }\end{array}$ & $14.5 \mathrm{~cm}$ \\
\hline $\mathrm{R}_{6}$ & $\begin{array}{l}\text { Outer radius of the magnetic } \\
\text { yoke }\end{array}$ & $18 \mathrm{~cm}$ \\
\hline $\mathrm{R}_{7}$ & Outer radius of the exterior & $20 \mathrm{~cm}$ \\
\hline $\mathrm{L}$ & Axial length of the motor & $100 \mathrm{~cm}$ \\
\hline$\beta$ & HTS bulk opening & $\pi / \mathrm{Q}$ \\
\hline$\alpha$ & Winding opening & $11 / 12$ \\
\hline $\mathrm{J}_{0}$ & $\begin{array}{l}\text { Maximum current density in } \\
\text { one phase windings }\end{array}$ & $60 \mathrm{~A} / \mathrm{mm}^{2}$ \\
\hline $\mathrm{p}$ & Pole-pairs number & 2 \\
\hline Q & Number of bulks (with $\mathrm{p}=\mathrm{Q} / 2$ ) & 4 \\
\hline $\mathrm{N}$ & $\begin{array}{l}\text { Number of harmonics used for } \\
\text { the magnetic field calculation } \\
\text { in the hole domains }\end{array}$ & 80 \\
\hline $\mathrm{K}$ & $\begin{array}{l}\text { Number of harmonics used for } \\
\text { the magnetic field calculation } \\
\text { in the others domains (It could } \\
\text { be different in each domain) }\end{array}$ & 100 \\
\hline
\end{tabular}

\section{Flux density distribution}

The magnetic flux lines under no-load and load conditions are shown respectively in figure 11a and figure 11b. The HTS bulks are represented in red color. The three-phase stator windings are fed with electrical current such as $I_{a}=I$ and $I_{b}=I_{c}=-I / 2$ corresponding to $\mathrm{AC}$ operation. The position angle of the rotor is fixed to $\theta_{0}=0^{\circ}$ for no-load condition and $\theta_{0}=22.5^{\circ}$ for load condition (that corresponds to the maximum torque position). Due to the diamagnetic behavior of the HTS bulk, the magnetic field lines are deviated around the superconducting bulks. The deviation of the field lines in the air-gap is the cause of the electromagnetic torque.

The corresponding flux density distributions (radial and tangential components) in the middle of the air-gap (at $r$ $=9.75 \mathrm{~cm})$ under no-load condition $\left(\theta_{0}=0^{\circ}\right)$ and load condition $\left(\theta_{0}=22.5^{\circ}\right)$ are plotted, respectively, in figure 13 and figure 14. The effect of the HTS bulks on the flux density waveforms is very clear. One can see the distortion of the flux density distribution at the location of the HTS bulks. The radial flux density is almost null behind the bulks due to the diamagnetic behavior of the superconducting material.

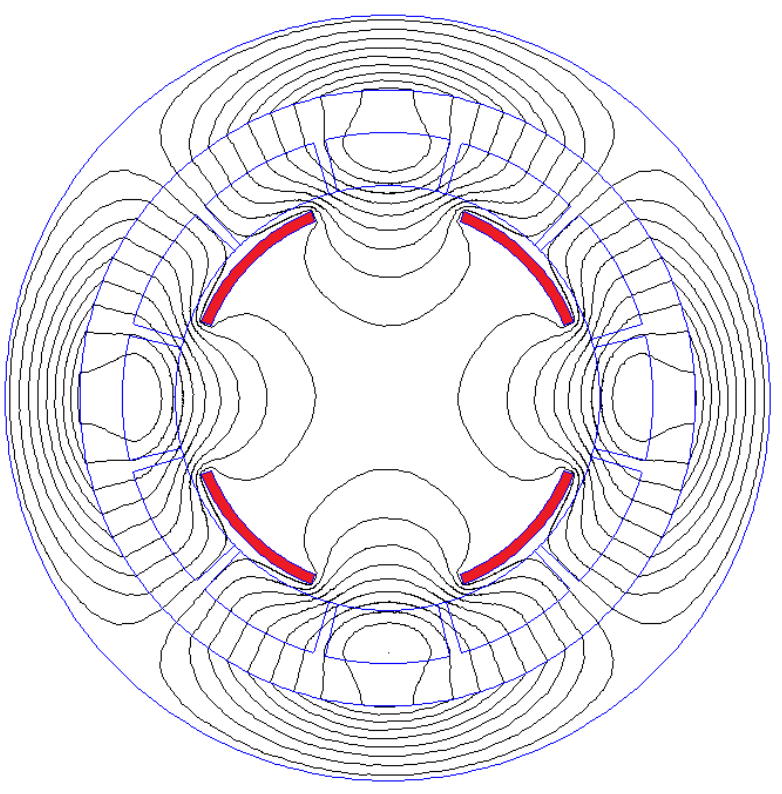

(a)

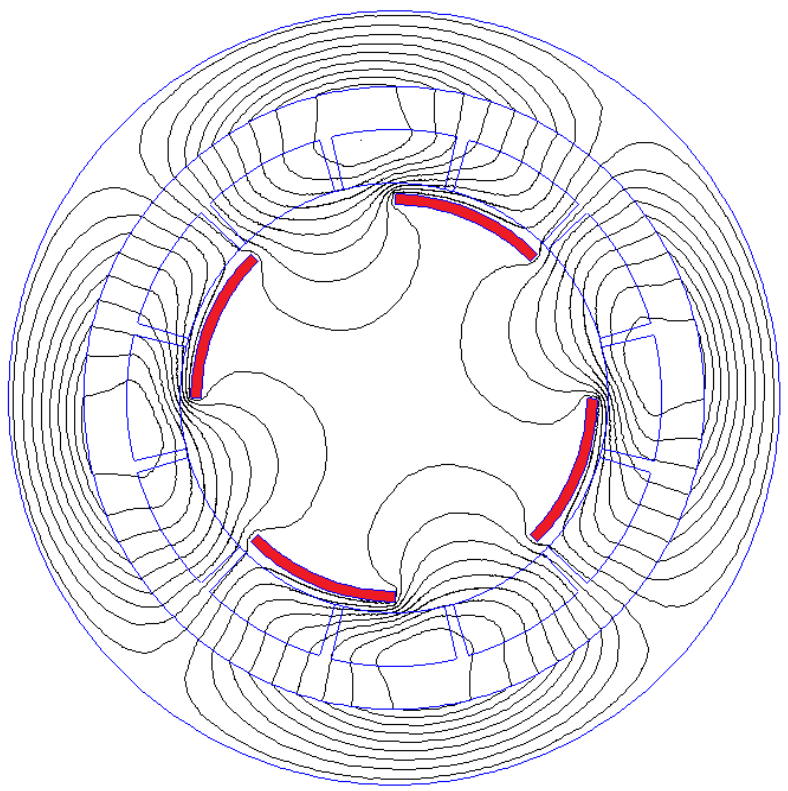

(b)

Figure 11. Magnetic flux lines under (a): no-load condition $\left(\theta_{0}=0^{\circ}\right)$ and $(\mathrm{b})$ : load condition $\left(\theta_{0}=22.5^{\circ}\right)$.

An excellent agreement with the results deduced from FEM is obtained. Figure 14 shows the flux density distribution for radial and tangential component in the middle of a hole domain (between HTS bulks). As expected, one can observe that the tangential flux density is null on the sides of the HTS bulks. The analytical and numerical results are again in close agreement. 


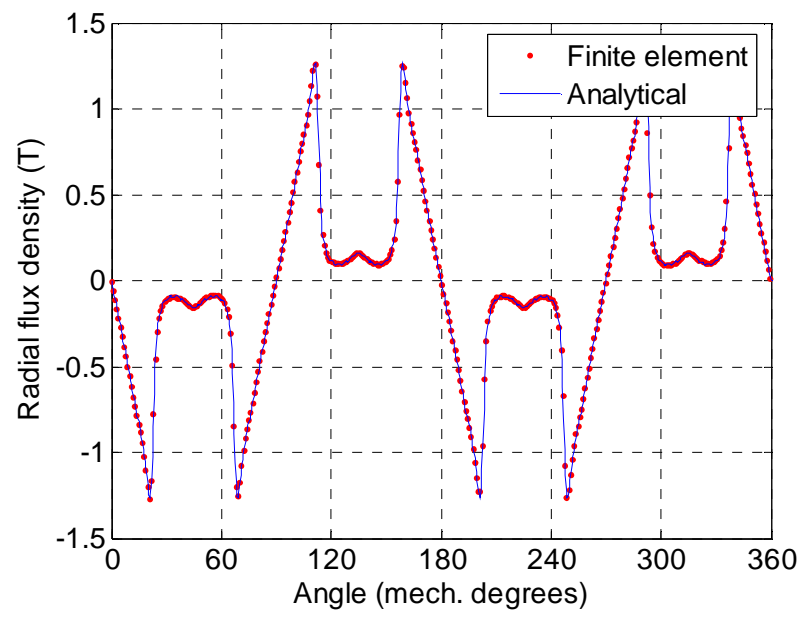

(a)

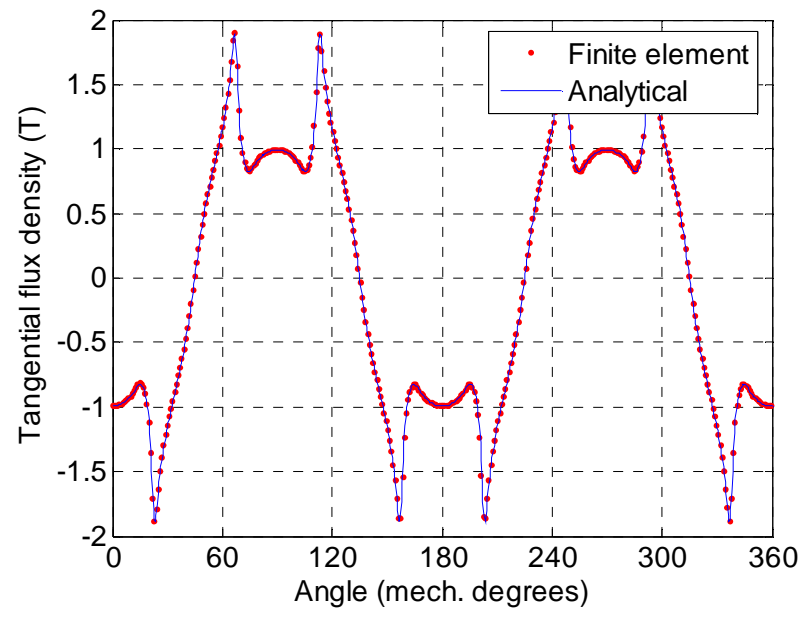

(b)

Figure 12. Flux density distribution for radial (a) and tangential (b) component in the middle of the airgap domain under no-load condition $\left(\theta_{0}=0^{\circ}\right)$.

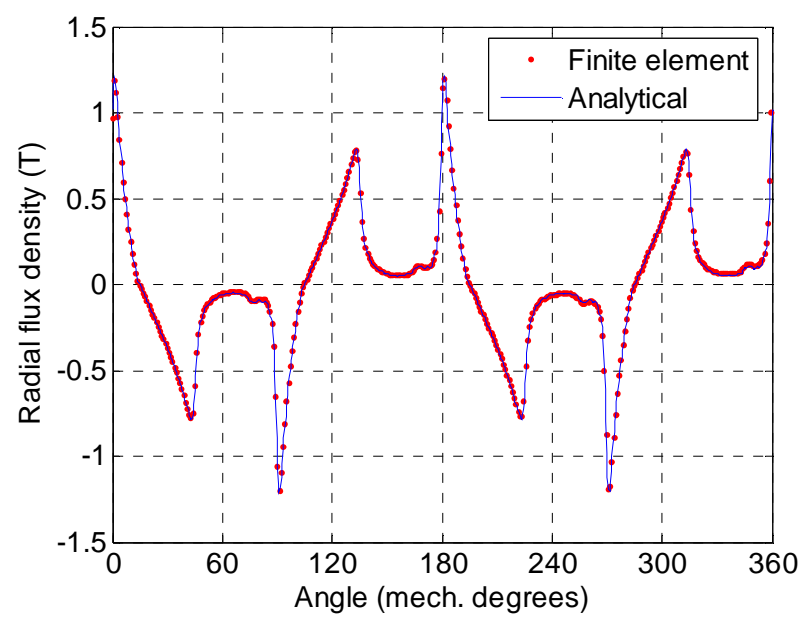

(a)

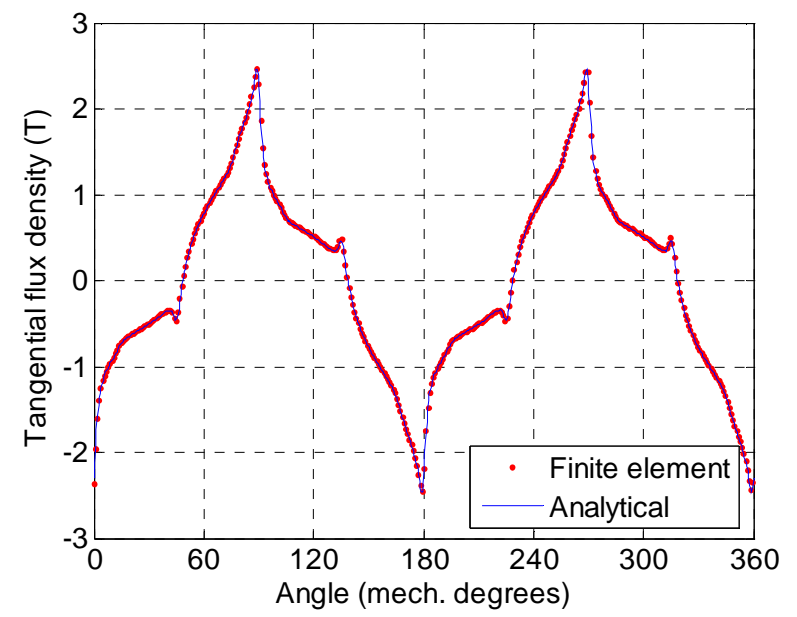

(b)

Figure 13. Flux density distribution for radial (a) and tangential (b) component in the middle of the airgap under load condition $\left(\theta_{0}=22.5^{\circ}\right)$

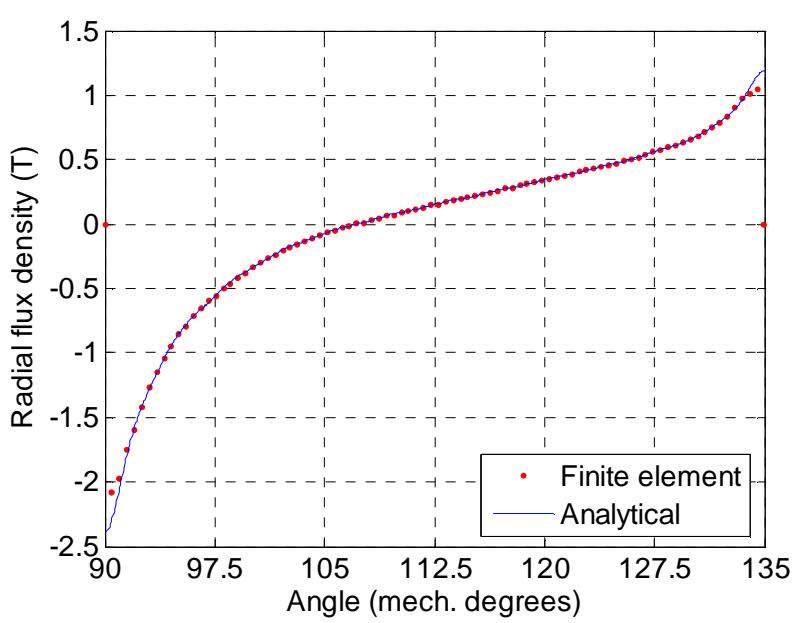

(a)

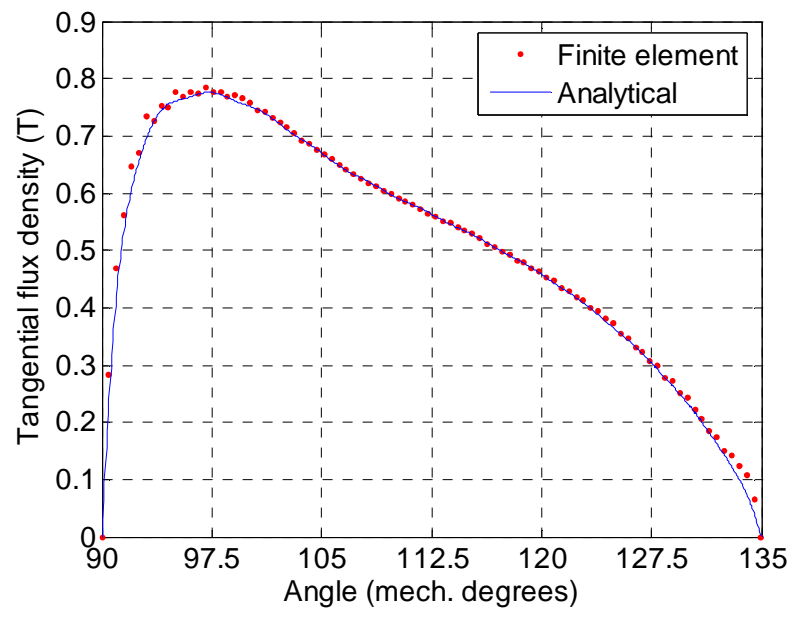

(b)

Figure 14. Flux density distribution for radial (a) and tangential (b) component in the middle of the hole domain under load condition $\left(\theta_{0}=22.5^{\circ}\right)$ 


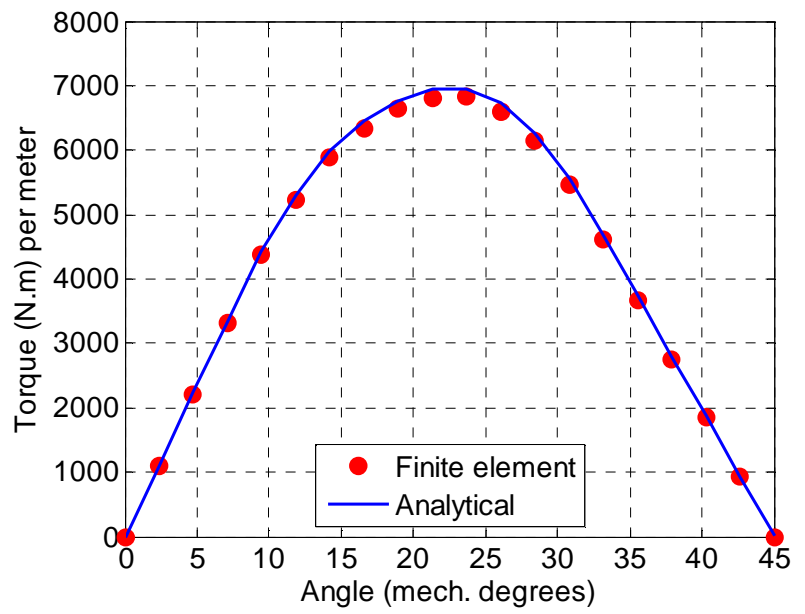

Figure 15. Static torque versus rotor position for $J_{0}=60 \mathrm{~A} / \mathrm{mm}^{2}$.

\section{Electromagnetic Torque}

The static torque versus mechanical rotor position $\theta_{0}$ is presented in figure 15. The three-phase HTS windings are fed with electrical current such as $I_{a}=I$ and $I_{b}=I_{c}=$ $I / 2$ corresponding to AC operation. Compared to the FE simulations, one can see that the analytical calculation well tracks the electromagnetic torque. The torque characteristic shown in figure 15 presents the same behavior of the classical result obtained for a conventional 4-pole Synchronous Reluctance Motor [35]. One can observe that a maximum torque of around $7000 \mathrm{~N}$.m is obtained for a value of $\theta_{0}$ equal to $22.5^{\circ}$.

Figure 16 shows the electromagnetic torque waveforms versus rotor position. The phase current waveforms in the three-phase HTS windings are sinusoidal. The studied machine produced an average torque of about 6800 N.m. It can be seen that the machine exhibits important torque ripple mainly due to the winding distribution. Once again, the analytical results are in good agreement with the FEM results.

\section{Conclusion}

In this paper, an analytical method to compute the airgap field distribution in a wholly HTS reluctance motor has been presented. The Laplace's and Poisson's equations in polar coordinates have been solved by the technique of separation of variables in the different subdomains (holes, air-gap, iron and windings). The solutions have been obtained using boundary and continuity conditions between the different subdomains.

The different results presented in this paper have shown that the proposed analytical model is able to predict the



Figure 16. Electromagnetic torque versus rotor position.

performance of the proposed HTS motor with a very good precision. Moreover, the analytical model presents less computational time consuming than numerical ones (finite element method). Hence, the analytical model developed in this paper can be used as a preliminary tool to investigate the influence of the design parameters such as HTS bulks opening, pole-pairs number, winding configurations. That will be done in future works.

\section{Appendix}

For the determination of the integration coefficients, integrals of the form have been calculate as following

$$
\begin{gathered}
f_{u}(n, i)=\int_{\theta_{i}}^{\theta_{i}+\beta} \cos (u \cdot \theta) \cdot \sin \left(\frac{n . \pi}{\beta}\left(\theta-\theta_{i}\right)\right) \cdot d \theta \\
g_{u}(n, i)=\int_{\theta_{i}}^{\theta_{i}+\beta} \sin (u . \theta) \cdot \sin \left(\frac{n \pi}{\beta}\left(\theta-\theta_{i}\right)\right) \cdot d \theta \\
N_{n}=\int_{\theta_{i}}^{\theta_{i}+\beta} \sin \left(\frac{n . \pi}{\beta}\left(\theta-\theta_{i}\right)\right) \cdot d \theta
\end{gathered}
$$

The development of (A.1) and (A.2), which are used in the expressions of the integration coefficients, are given by

$$
\begin{aligned}
& \text { - for } n \pi \neq u \beta \\
& f_{u}(n, i)=\frac{n \pi \beta}{(u \beta)^{2}-(n \pi)^{2}}\left((-1)^{n} \cos \left(\left(\theta_{i}+\beta\right) u\right)-\cos \left(\theta_{i} u\right)\right)
\end{aligned}
$$


$g_{u}(n, i)=\frac{n \pi \beta}{(u \beta)^{2}-(n \pi)^{2}}\left((-1)^{n} \sin \left(\left(\theta_{i}+\beta\right) u\right)-\sin \left(\theta_{i} u\right)\right)$

- for $n \pi=u \beta$

$f_{u}(n, i)=-\frac{\beta}{2}\left(\sin \left(u \theta_{i}\right)+\frac{1}{2 n \pi}\left(\cos \left(\left(\theta_{i}+2 \beta\right) u\right)-\cos \left(\theta_{i} u\right)\right)\right)$

$g_{u}(n, i)=\frac{\beta}{2}\left(\cos \left(u \theta_{i}\right)-\frac{1}{2 n \pi}\left(\sin \left(\left(\theta_{i}+2 \beta\right) u\right)-\sin \left(\theta_{i} u\right)\right)\right)$

The development of (A.3) gives the following functions

$$
N_{n}=\beta \frac{1-(-1)^{n}}{n \pi}
$$

- Expressions of the coefficients $A_{0}^{I}, A_{h}^{I}$ and $C_{h}^{I}$ for the rotor shaft sub-domain

The development of (28), (29) and (30) gives

$$
\begin{aligned}
& A_{0}^{I}=\frac{1}{2 . \pi} \sum_{i=1}^{Q} \sum_{n=1}^{\infty} C_{n}^{i} \frac{\beta \cdot R_{1}}{n \cdot \pi} \frac{\mathrm{P}_{n \pi / \beta}\left(R_{1}, R_{2}\right)}{\mathrm{E}_{n \pi / \beta}\left(R_{1}, R_{2}\right)} \cdot N_{n} \\
& +\frac{1}{2 . \pi} \sum_{i=1}^{Q} \sum_{n=1}^{\infty} D_{n}^{i} \frac{\beta \cdot R_{2}}{n \cdot \pi} \frac{2}{\mathrm{E}_{n \pi / \beta}\left(R_{2}, R_{1}\right)} \cdot N_{n} \\
& A_{h}^{I}=\frac{1}{\pi} \sum_{i=1}^{Q} \sum_{n=1}^{\infty} C_{n}^{i} \frac{\beta \cdot R_{1}}{n \cdot \pi} \frac{\mathrm{P}_{n \pi / \beta}\left(R_{1}, R_{2}\right)}{\mathrm{E}_{n \pi / \beta}\left(R_{1}, R_{2}\right)} \cdot f_{u}(n, i) \\
& +\frac{1}{\pi} \sum_{i=1}^{Q} \sum_{n=1}^{\infty} D_{n}^{i} \frac{\beta \cdot R_{2}}{n \cdot \pi} \frac{2}{\mathrm{E}_{n \pi / \beta}\left(R_{2}, R_{1}\right)} \cdot f_{u}(n, i) \\
& C_{h}^{I}=\frac{1}{\pi} \sum_{i=1}^{Q} \sum_{n=1}^{\infty} C_{n}^{i} \frac{\beta \cdot R_{1}}{n \cdot \pi} \frac{\mathrm{P}_{n \pi / \beta}\left(R_{1}, R_{2}\right)}{\mathrm{E}_{n \pi / \beta}\left(R_{1}, R_{2}\right)} \cdot g_{u}(n, i) \\
& +\frac{1}{\pi} \sum_{i=1}^{Q} \sum_{n=1}^{\infty} D_{n}^{i} \frac{\beta \cdot R_{2}}{n \cdot \pi} \frac{2}{\mathrm{E}_{n \pi / \beta}\left(R_{2}, R_{1}\right)} \cdot g_{u}(n, i)
\end{aligned}
$$

- Expressions $A_{0}^{I I I}, B_{0}^{I I I}, A_{k}^{I I I}, B_{k}^{I I I}, C_{k}^{I I I}$ and $D_{k}^{I I I}$ for the windings sub-domain.

The development of (80), (81), (82), (83), (84) and (85) gives

$$
\begin{aligned}
& A_{0}^{I I I}=A_{0}^{I I}+B_{0}^{I I} \cdot \ln \left(R_{2} / R_{3}\right) \\
& B_{0}^{I I I}=A_{0}^{I V}+B_{0}^{I V} \cdot \ln \left(R_{5} / R_{4}\right)
\end{aligned}
$$

$A_{k}^{I I I}=\sum_{j=1}^{\infty}\left(A_{j}^{I I} \frac{R_{3}}{j} \frac{\mathrm{E}_{j}\left(R_{3}, R_{2}\right)}{P_{j}\left(R_{3}, R_{2}\right)}+B_{j}^{I I} \frac{2}{P_{j}\left(R_{2}, R_{3}\right)}\right)$

$$
B_{k}^{I I I}=\sum_{l=1}^{\infty}\left(A_{l}^{I V} \frac{R_{4}}{l} \frac{\mathrm{E}_{l}\left(R_{4}, R_{5}\right)}{P_{l}\left(R_{4}, R_{5}\right)}+B_{l}^{I V} \frac{2}{P_{l}\left(R_{5}, R_{4}\right)}\right)
$$

$$
C_{k}^{I I I}=\sum_{j=1}^{\infty}\left(C_{j}^{I I} \frac{R_{3}}{j} \frac{\mathrm{E}_{j}\left(R_{3}, R_{2}\right)}{P_{j}\left(R_{3}, R_{2}\right)}+D_{j}^{I I} \frac{2}{P_{j}\left(R_{2}, R_{3}\right)}\right)
$$

$$
D_{k}^{I I I}=\sum_{l=1}^{\infty}\left(C_{l}^{I V} \frac{R_{4}}{l} \frac{\mathrm{E}_{l}\left(R_{4}, R_{5}\right)}{P_{l}\left(R_{4}, R_{5}\right)}+D_{l}^{I V} \frac{2}{P_{l}\left(R_{5}, R_{4}\right)}\right)
$$

- Expressions $A_{0}^{I I}, B_{0}^{I I}, A_{j}^{I I}, B_{j}^{I I}, C_{j}^{I I}$ and $D_{j}^{I I}$ for the air-gap sub-domain.

The development of (36), (37), (38), (39), (40) and (41) gives

$$
\begin{gathered}
A_{0}^{I I}=\frac{1}{2 \cdot \pi} \cdot \sum_{i=1}^{Q} \sum_{n=1}^{\infty} C_{n}^{i} \cdot \frac{\beta \cdot R_{1}}{n \cdot \pi} \frac{2}{\mathrm{E}_{n \pi / \beta}\left(R_{1}, R_{2}\right)} \cdot N_{n} \\
+\frac{1}{2 \cdot \pi} \cdot \sum_{i=1}^{Q} \sum_{n=1}^{\infty} D_{n}^{i} \cdot \frac{\beta \cdot R_{2}}{n \cdot \pi} \frac{\mathrm{P}_{n \pi / \beta}\left(R_{2}, R_{1}\right)}{\mathrm{E}_{n \pi / \beta}\left(R_{2}, R_{1}\right)} \cdot N_{n} \\
B_{0}^{I I}=\left(\frac{A_{0}^{I I I}-B_{0}^{I I I}}{\ln \left(R_{3} / R_{4}\right)}\right)
\end{gathered}
$$

$A_{j}^{I I}=\sum_{k=1}^{\infty} \frac{k}{R_{3}} \cdot A_{k}^{I I I} \cdot \frac{P_{k}\left(R_{3}, R_{4}\right)}{\mathrm{E}_{k}\left(R_{3}, R_{4}\right)}$

$+\sum_{k=1}^{\infty} \frac{k}{R_{3}} \cdot B_{k}^{I I I} \cdot \frac{2}{\mathrm{E}_{k}\left(R_{4}, R_{3}\right)}+\sum_{k=1}^{\infty} G_{k}^{\prime}\left(R_{3}\right) \cdot \cos \left(\varphi_{1}\right)$

$B_{j}^{I I}=\frac{1}{\pi} \cdot \sum_{i=1}^{Q} \sum_{n=1}^{\infty} C_{n}^{i} \cdot \frac{\beta \cdot R_{1}}{n \cdot \pi} \frac{2}{\mathrm{E}_{n \pi / \beta}\left(R_{1}, R_{2}\right)} \cdot f_{u}(n, i)$

$+\frac{1}{\pi} \cdot \sum_{i=1}^{Q} \sum_{n=1}^{\infty} D_{n}^{i} \cdot \frac{\beta \cdot R_{2}}{n \cdot \pi} \frac{\mathrm{P}_{n \pi / \beta}\left(R_{2}, R_{1}\right)}{\mathrm{E}_{n \pi / \beta}\left(R_{2}, R_{1}\right)} \cdot f_{u}(n, i)$

$C_{j}^{I I}=\sum_{k=1}^{\infty} \frac{k}{R_{3}} \cdot C_{k}^{I I I} \cdot \frac{P_{k}\left(R_{3}, R_{4}\right)}{\mathrm{E}_{k}\left(R_{3}, R_{4}\right)}$

$+\sum_{k=1}^{\infty} \frac{k}{R_{3}} \cdot D_{k}^{I I I} \cdot \frac{2}{\mathrm{E}_{k}\left(R_{4}, R_{3}\right)}+\sum_{k=1}^{\infty} G_{k}^{\prime}(R 3) \cdot \sin \left(\varphi_{1}\right)$ 
$D_{j}^{I I}=\frac{1}{\pi} \sum_{i=1}^{Q} \sum_{n=1}^{\infty} C_{n}^{i} \frac{\beta \cdot R_{1}}{n \cdot \pi} \frac{2}{\mathrm{E}_{n \pi / \beta}\left(R_{1}, R_{2}\right)} \cdot g_{u}(n, i)$

$+\frac{1}{\pi} \sum_{i=1}^{Q} \sum_{n=1}^{\infty} D_{n}^{i} \frac{\beta . R_{2}}{n \cdot \pi} \frac{\mathrm{P}_{n \pi / \beta}\left(R_{2}, R_{1}\right)}{\mathrm{E}_{n \pi / \beta}\left(R_{2}, R_{1}\right)} \cdot g_{u}(n, i)$

where $Q$ is the number of rotor holes and $G_{k}^{\prime}\left(R_{3}\right)=\left.\frac{\partial G_{k}(r)}{\partial r}\right|_{r=R_{3}}$.

- Expressions $A_{0}^{I V}, B_{0}^{I V}, A_{l}^{I V}, B_{l}^{I V}, C_{l}^{I V}$ and $D_{l}^{I V}$ for the outer air-gap sub-domain.

The development of (50), (51), (52), (53), (54) and (55) gives

$$
\begin{gathered}
A_{0}^{I V}=A_{0}^{V}+B_{0}^{V} \cdot \ln \left(R_{6} / R_{5}\right) \\
B_{0}^{I V}=\left(\frac{A_{0}^{I I I}-B_{0}^{I I I}}{\ln \left(R_{3} / R_{4}\right)}\right) \\
A_{l}^{I V}=\sum_{k=1}^{\infty} \frac{k}{R_{4}} A_{k}^{I I I} \frac{2}{\mathrm{E}_{k}\left(R_{3}, R_{4}\right)} \\
\left.+\sum_{k=1}^{\infty} \frac{k}{R_{4}} B_{k}^{I I I} \frac{P_{k}\left(R_{4}, R_{3}\right)}{\mathrm{E}_{k}\left(R_{4}, R_{3}\right)}+\sum_{k=1}^{\infty} G_{k}^{\prime}(R 4)\right) \cdot \cos \left(\varphi_{1}\right) \\
B_{l}^{I V}=\sum_{f=1}^{\infty}\left(A_{f}^{V} \frac{R_{5}}{f} \frac{\mathrm{E}_{f}\left(R_{5}, R_{6}\right)}{P_{f}\left(R_{5}, R_{6}\right)}+B_{f}^{V} \frac{2}{P_{f}\left(R_{6}, R_{5}\right)}\right) \\
C_{l}^{I V}=\sum_{k=1}^{\infty} \frac{k}{R_{4}} C_{k}^{I I I} \frac{2}{\mathrm{E}_{k}\left(R_{3}, R_{4}\right)} \\
\left.+\sum_{k=1}^{\infty} \frac{k}{R_{4}} D_{k}^{I I I} \frac{P_{k}\left(R_{4}, R_{3}\right)}{\mathrm{E}_{k}\left(R_{4}, R_{3}\right)}+\sum_{k=1}^{\infty} G_{k}^{\prime}(R 4)\right) \cdot \sin \left(\varphi_{1}\right) \\
D_{l}^{I V}=\sum_{f=1}^{\infty}\left(C_{f}^{V} \frac{R_{5}}{f} \frac{\mathrm{E}_{f}\left(R_{5}, R_{6}\right)}{P_{f}\left(R_{5}, R_{6}\right)}+D_{f}^{V} \frac{2}{P_{f}\left(R_{6}, R_{5}\right)}\right)
\end{gathered}
$$

where $G_{k}^{\prime}\left(R_{4}\right)=\left.\frac{\partial G_{k}(r)}{\partial r}\right|_{r=R_{4}}$.

- Expressions $A_{0}^{V}, B_{0}^{V}, A_{f}^{V}, B_{f}^{V}, C_{f}^{V}$ and $D_{f}^{V}$ for the magnetic yoke sub-domain.

The development of (60), (61), (62), (63), (64) and (65) gives

$$
A_{0}^{V}=B_{0}^{V I} \cdot \ln \left(R_{7} / R_{6}\right)
$$

$$
\begin{gathered}
B_{0}^{V}=-\mu_{r} \cdot B_{0}^{I V} \\
A_{f}^{V}=\mu_{r} \sum_{l=1}^{\infty}\left(A_{l}^{I V} \frac{R_{4}}{R_{5}} \frac{2}{P_{l}\left(R_{4}, R_{5}\right)}+B_{l}^{I V} \frac{l}{R_{5}} \frac{E_{l}\left(R_{5}, R_{4}\right)}{P_{l}\left(R_{5}, R_{4}\right)}\right) \\
B_{f}^{V}=\sum_{m=1}^{\infty}\left(A_{m}^{I V} \frac{R_{6}}{m} \frac{\mathrm{E}_{m}\left(R_{6}, R_{7}\right)}{P_{m}\left(R_{6}, R_{7}\right)}\right) \\
C_{f}^{V}=\mu_{r} \sum_{l=1}^{\infty}\left(C_{l}^{I V} \frac{R_{4}}{R_{5}} \frac{2}{P_{l}\left(R_{4}, R_{5}\right)}+D_{l}^{I V} \frac{l}{R_{5}} \frac{E_{l}\left(R_{5}, R_{4}\right)}{P_{l}\left(R_{5}, R_{4}\right)}\right) \\
D_{f}^{V}=\sum_{m=1}^{\infty}\left(C_{m}^{I V} \frac{R_{6}}{m} \frac{\mathrm{E}_{m}\left(R_{6}, R_{7}\right)}{P_{m}\left(R_{6}, R_{7}\right)}\right)
\end{gathered}
$$

- Expressions $B_{0}^{V I}, A_{m}^{V I}$ and $C_{m}^{V I}$ for the exterior subdomain.

The development of (70), (71) and (72) gives

$$
\begin{gathered}
B_{0}^{V I}=-\frac{B_{0}^{V}}{\mu_{r}} \\
A_{m}^{V I}=\frac{1}{\mu_{r}} \sum_{f=1}^{\infty}\left(A_{f}^{V} \frac{R_{5}}{R_{6}} \frac{2}{P_{f}\left(R_{5}, R_{6}\right)}+B_{f}^{V} \frac{f}{R_{6}} \frac{E_{f}\left(R_{6}, R_{5}\right)}{P_{f}\left(R_{6}, R_{5}\right)}\right) \\
C_{m}^{V I}=\frac{1}{\mu_{r}} \sum_{f=1}^{\infty}\left(C_{f}^{V} \frac{R_{5}}{R_{6}} \frac{2}{P_{f}\left(R_{5}, R_{6}\right)}+D_{f}^{V} \frac{f}{R_{6}} \frac{E_{f}\left(R_{6}, R_{5}\right)}{P_{f}\left(R_{6}, R_{5}\right)}\right)
\end{gathered}
$$

- Expressions $C_{n}^{i}$ and $D_{n}^{i}$ for the i-th hole sub-domain.

The development of (19) and (20) gives

$$
C_{n}^{i}=\frac{2}{\beta} \sum_{h=1}^{\infty}\left(A_{h}^{I} \frac{h}{R_{1}} \cdot f_{u}(n, i)+C_{h}^{I} \frac{h}{R_{1}} \cdot g_{u}(n, i)\right)
$$

$$
D_{n}^{i}=-\frac{B_{0}^{I I} \cdot N_{n}}{R_{2}} \cdot \frac{2}{\beta}
$$

$+\frac{2}{\beta} \sum_{j=1}^{\infty}\left(A_{j}^{I I} \frac{R_{3}}{R_{2}} \frac{2}{P_{j}\left(R_{3}, R_{2}\right)}+B_{j}^{I I} \frac{j}{R_{2}} \frac{E_{j}\left(R_{2}, R_{3}\right)}{P_{j}\left(R_{2}, R_{3}\right)}\right) \cdot f_{u}(n, i)$

$+\frac{2}{\beta} \sum_{j=1}^{\infty}\left(C_{j}^{I I} \frac{R_{3}}{R_{2}} \frac{2}{P_{j}\left(R_{3}, R_{2}\right)}+D_{j}^{I I} \frac{j}{R_{2}} \frac{E_{j}\left(R_{2}, R_{3}\right)}{P_{j}\left(R_{2}, R_{3}\right)}\right) \cdot g_{u}(n, i)$ 
So a system of linear equations with the same number of unknowns will be solve. By rewriting the above equations in matrix and vectors format, a numerical solution can be found by using mathematical software (Matlab).

\section{References}

[1] Farlow S J 1993 Partial Differential Equations for Scientists and Engineers (New York: Dover)

[2] Meeker D C Finite Element Method Magnetics Version 4.2 (1 April 2009 Build), http://www.femm.info

[3] Gu Q and Gao H 1985 Effect of slotting in PM electrical machines Elect. Mach. Power Syst. 10 273-84

[4] Lubin T, Mezani S and Rezzoug A 2010 Exact analytical method for magnetic field computation in the air-gap of cylindrical electrical machines considering slotting effects IEEE Trans. Magn. 46 1092-99

[5] Liu Z J, Li J T, and Jiang Q 2008 An improved analytical solution for predicting magnetic forces in permanent magnet motors J. Appl.Phys. 103

[6] Zhu Z Q, Wu L J and Xia Z P 2010 An accurate subdomain model for magnetic field computation in slotted surface-mounted permanent magnet machines IEEE Trans. Magn. 46 1100-15

[7] Gysen B L J, Meessen K J, Paulides J J H and Lomonova E A 2010 General formulation of the electromagnetic field distribution in machines and devices using Fourier analysis IEEE Trans. Magn. 46 39-52

[8] Schiferl R, Flory A, Livoti W C and Umans S D 2008 High-Temperature Superconducting Synchronous Motors: Economic Issues for Industrial Applications IEEE Trans. Appl. Superconduct. 44 1376-84

[9] Chu S D, Torii S 2005 Torque-speed characteristics of superconducting synchronous reluctance motors with DyBCO bulk in the rotor IEEE Trans. Appl. Superconduct. 15 2178-81

[10] Kovalev L K, Ilushin K V, Koneev S M-A, Kovalev K L, Penkin V T, Poltavets V N, Gawalek W, Habisreuther T, Oswald B and Best K -J 1999 Hysteresis and reluctance electric machines with bulk HTS rotor elements IEEE Trans. Appl. Superconduct. 9 1261-64

[11] Kovalev L K, Ilushin K V, Penkin V T, Kovalev K L, Koneev S M-A, Poltavets V N, Larionoff A E, Modestov K A, Larionoff S A, Gawalek W, Habisreuther T, Oswald B, Best K-J and Strasser T 2000 Hysteresis and reluctance electric machines with bulk HTS elements. Recent results and future development IOP Supercond. Sci. Technol. 13 498-502

[12] Barnes G J, McCulloch M D and Dew-Hughes D 2000 Applications and modelling of bulk HTSs in brushless ac machines IOP Supercond. Sci. Technol. 17 875-78

[13] Oswald B, Best K -J, Setzer M, Söll M, Gawalek W, Gutt A, Kovalev L, Krabbes G, Fisher L and Freyhardt H C 2005 Reluctance motors with bulk HTS material IOP Supercond. Sci. Technol. 18 24-29 